\title{
A Computational Investigation for Determining the Natural Frequencies and Damping Effects of Diaphragm- Implemented Spacecraft Propellant Tanks
}

\author{
Brian Lenahen ${ }^{1}$, Adrien Bernier ${ }^{2}$ and Sathya Gangadharan ${ }^{3}$ \\ Embry-Riddle Aeronautical University, 600 South Clyde Morris Blvd., Daytona Beach, FL, 32114 \\ James Sudermann ${ }^{4}$ and Brandon Marsell ${ }^{5}$ \\ NASA, Kennedy Space Center, FL, 32899
}

\begin{abstract}
Spin-stabilization maneuvers are typically performed by spacecraft entering low-earth orbit to maintain attitude stability. These maneuvers induce periodic fluid movement inside the spacecraft's propellant tank known as fuel slosh, which is responsible for creating forces and moments on the sidewalls of the propellant tank. These forces and moments adversely affect spin-stabilization and risk jeopardizing the mission of the spacecraft. Therefore, propellant tanks are designed with propellant management devices (PMD's) such as baffles or diaphragms which work to counteract the forces and moments associated with fuel slosh. However, despite the presence of PMD's, the threat of spin-stabilization interference still exists should the propellant tank be excited at its natural frequency. When the fluid is excited at its natural frequency, the forces and moments acting on the propellant tank are amplified and may result in destabilizing the spacecraft. Thus, a computational analysis is conducted concerning diaphragm-implemented propellant tanks excited at their natural frequencies. Using multi-disciplinary computational fluid dynamics (CFD) software, computational models are developed to reflect potential scenarios that spacecraft propellant tanks could experience. By simulating the propellant tank under a wide array of parameters and variables including fill-level, gravity and diaphragm material and shape, a better understanding is gained as to how these parameters individually and collectively affect liquid propellant tanks and ultimately, spacecraft attitude dynamics.
\end{abstract}

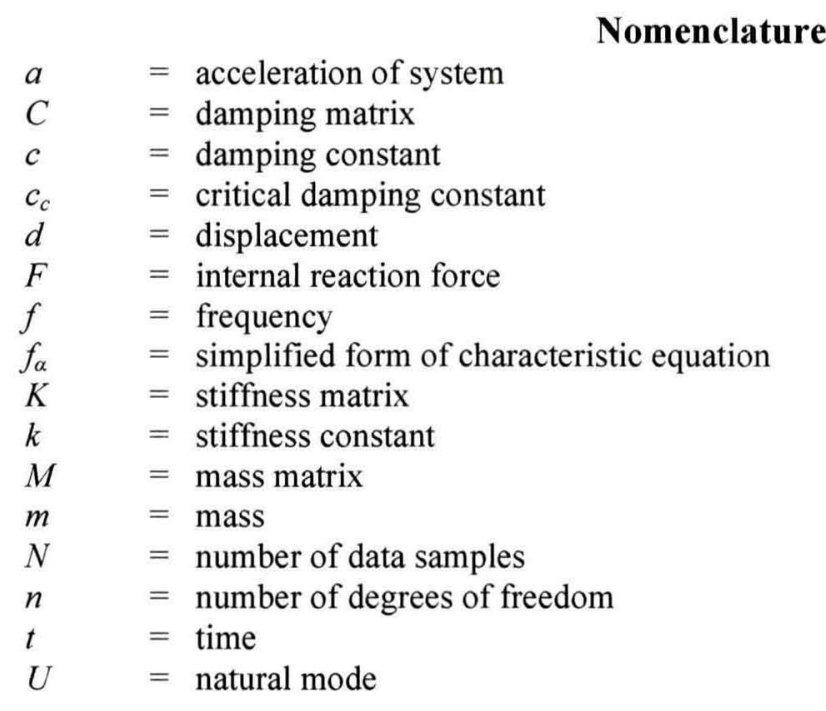

\footnotetext{
${ }^{1}$ Graduate Student, Department of Mechanical Engineering, AIAA Student Member

${ }^{2}$ Graduate Intern, Department of Mechanical Engineering

${ }^{3}$ Professor, Department of Mechanical Engineering, AIAA Associate Fellow

${ }_{5}^{4}$ Controls Analyst, NASA Launch Services Program

${ }^{5}$ Thermal/Fluids Analyst, QinetiQ North America, Launch Services Program, AIAA Member
} 


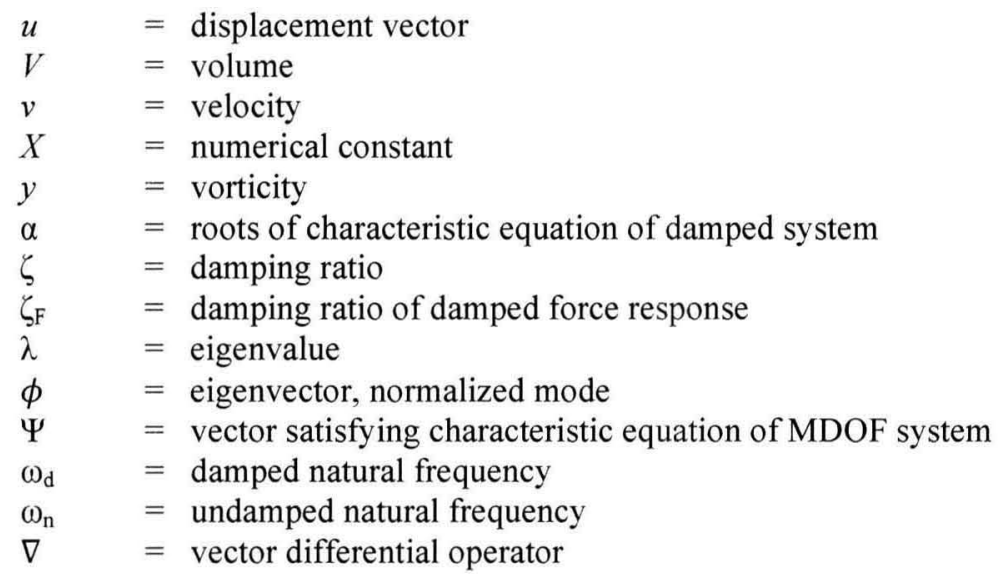

\section{Introduction}

The unsteady motion of liquid propellant and its effects on spin-stabilized space vehicles is of utmost concern when considering the attitude stability of the space vehicle as a whole. Spin stabilization maneuvers performed by spacecraft are intended to maintain proper trajectory of the spacecraft as it enters into an orbit. However, these maneuvers induce periodic oscillations of fluid movement on the surface of the propellant known as fuel slosh. This fluid movement dissipates energy on the molecular level in the form of small forces and moments on the sidewalls of the propellant tank. These adverse forces and moments interfere with the attitude control system of the vehicle and threaten to compromise the spacecraft's mission. Additionally, should the liquid propellant become excited at its natural frequency, the forces and moments can significantly increase, further threatening the spinstabilization of the space vehicle. In order to counteract these adverse forces and moments, propellant tanks are designed with propellant management devices (PMD's), of which the most common are baffles and diaphragms.

Baffles and diaphragms each have their own advantages and disadvantages depending on the specific fuel slosh scenario. Baffles are most effective in dampening sub-surface wave excitation, a common disturbance in propellant tanks excited at or near a $100 \%$ fill-level. Diaphragms are most effective in dampening surface waves as the diaphragm couples to the surface of the propellant, adapting shape as the fluid sloshes. This research is concerned with the effects of surface slosh on propellant tanks excited at their natural frequencies. Thus, all computational models are implemented with a diaphragm PMD to dampen fluid slosh.

Current research is aimed at investigating the benefits of using alternative diaphragm materials to combat the effects of surface slosh. Most diaphragms are made from a flexible, rubber-like material that is able to adjust shape and remain coupled to the surface of the propellant at all fill-levels. However, as of recent, new technology is focused on the effects of more rigid diaphragms, known as metallic diaphragms, and their damping effects on surface slosh. These metallic diaphragms behave similar to the flexible diaphragms in so much as they adjust shape and position with propellant depletion, but do not remain entirely coupled to the fluid surface. The fact that metallic diaphragms do not couple to the liquid surface is an interesting parameter investigated in this research. Comparisons between the flexible and metallic diaphragm models are made to determine the extent to which the metallic diaphragms' uncoupled behavior with the liquid surface impacts fluid damping in relation to the damping induced from the flexible diaphragm's coupled behavior with the liquid surface.

In order to conduct a proper comparative analysis of flexible diaphragms and metallic diaphragms, certain parameters must be defined across all computational models. The most important parameter is the propellant filllevel. Past research suggests the most critical fill-level is that of $60 \%$ full, for it is at this fill-level that forces and moments experienced from surface slosh are the greatest. Additional parameters defined in the computational models include gravity, turbulence and diaphragm shape.

All computational models are simulated using ANSYS CFX, a multi-disciplinary computational fluid dynamics (CFD) software. Additionally, to simulate the fluid-structure interaction (FSI) between the flexible diaphragm and the propellant, a two-way coupled solver is established between the fluids solver, CFX, and the ANSYS transient structural solver. Using multi-field physics, MFX, ANSYS is able to create the necessary FSI to obtain a coupled solution. 
The intent of this research is to investigate the damping effects and fluid behavior of flexible and rigid diaphragms implemented on spherical propellant tanks, excited at their natural frequencies. Using the force vs. time outputs from the computational simulations, the natural frequencies and damping ratio of the systems can be determined through signal analysis. The damped natural frequency and the damping ratio are two critical parameters necessary for initial spacecraft design.

\section{Theoretical Approach}

The viscous damping force is a function of the damping constant, $c$, and the velocity of the excited system, $\dot{u}$. Working through the differential equations that result from the application of Newton's Law, the following equation is derived.

A solution in the form,

$$
m \ddot{u}+c \dot{u}+k u=0
$$

$$
u(t)=X e^{\alpha t}
$$

is assumed which results in the characteristic equation for damped systems.

$$
m \alpha^{2}+c \alpha+k=0
$$

The roots of this equation are as follows.

$$
\alpha_{1,2}=\frac{-c}{2 m} \pm \sqrt{\left(\frac{c}{2 m}\right)^{2}-\frac{k}{m}}
$$

The nature of the roots is dependent on the type of damping taking place: underdamping, overdamping or critical damping. Although the type of damping taking place within the system may not be readily clear or understood, the solution to the equation (the roots) lends insight as to what type of damping the system is experiencing. For the purposes of this research investigation, the test models are divided into two categories: low damping $(\zeta<10 \%)$ and high damping $(\zeta>10 \%)$.

At the start of this research investigation, various numerical methods are explored to determine which method is best suited to analyze the damping for a particular model. Methods which consider single degree of freedom (SDOF) systems lend insight into the basic damping parameters but this research is focused on achieving more accurate results through the implementation of numerical methods applied to multiple degrees of freedom (MDOF) systems. Where feasible and applicable, computational analysis will consider two degrees of freedom for numerical analysis. The specific degrees of freedom utilized are determined from the force response outputs from the computational software used to model the fuel slosh systems. The two largest force response outputs correspond to the degrees of freedom where the majority of the inertial loading is received as the system is excited. The data from these two force responses is used for numerical analysis and signal processing.

Simulation models that have very low damping within the system are not suited for real-world implementation but are rather ideal for numerical analysis. 


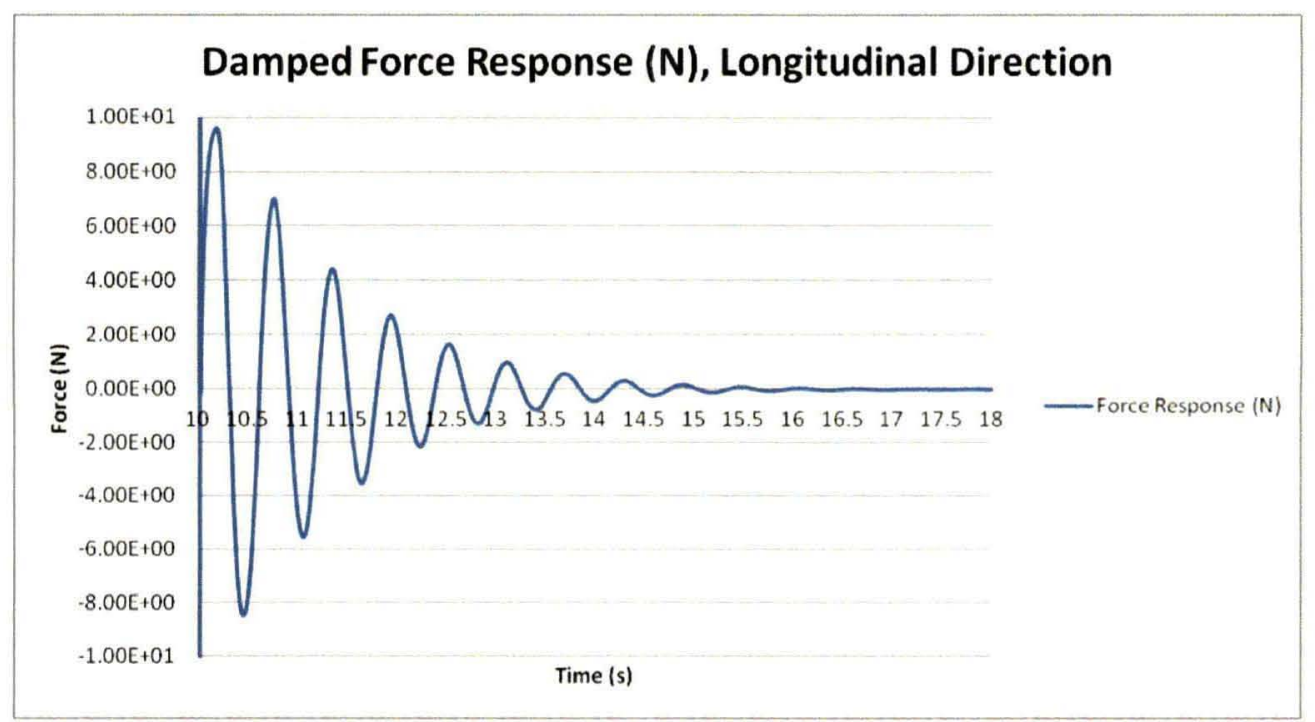

Figure 1. Damped Force Response Output, Low Damping

Figure 1 illustrates a damped force response of a system with low damping. The low damping allows many force response peaks to exist during the damping period of approximately 8 seconds. With this sufficient amount of data, more exact numerical methods can be used during analysis to extract the necessary damping parameters.

Frequency analysis of a discrete signal is best analyzed using a Discrete Fourier Transform (DFT) which transforms a signal from the time domain (as shown in Figure 1) into a signal represented in the frequency domain. However, the methods used to calculate the DFT are tedious as there are many complex multiplications and additions which must be performed to transform the signal into the frequency domain. Computationally, a quicker and just as effective method of frequency analysis is the Fast Fourier Transform (FFT) which does the exact same calculations as the DFT, but in much less time. The DFT requires $(N-1)^{2}$ complex multiplications and $N(N$ 1) complex additions as opposed to the FFT's approach of breaking the signal down into a series of 2 point samples which only require 1 multiplication and 2 additions and the recombination of the points which is minimal. ${ }^{2}$

The FFT calculations are performed in MATLAB from pre-defined algorithms in the software. The force response signal is divided into the response during excitation and the response during damping. ${ }^{i}$ The FFT is individually applied to these two signals and the resulting amplitude vs. frequency plot is output for the two signals respectively. Analyzing the FFT plot during excitation will yield the undamped natural frequency of the system, $\omega_{\mathrm{n}}$, while analyzing the FFT plot during damping will yield the damped natural frequency of the system, $\omega_{\mathrm{d}}$.

Common industry practice for analyzing fuel slosh force response data is the use of spectral analysis to obtain a spectrogram of the data. The spectrogram illustrates time as a function of frequency and is color-coded to signify the strength of the signal. In following standard practice and for the purposes of this research investigation, spectrograms are formulated to validate the results obtained from the FFT plots. Once the undamped and damped natural frequencies are confirmed to be accurate based off two unique and independent analyses, the damping constant, $c$, the critical damping constant, $c_{c}$, the stiffness, $k$, and the damping ratio, $\zeta$, of the system are calculated. ${ }^{1}$

The remaining damping parameter to be determined is the damping ratio, $\zeta_{\mathrm{F}}$, obtained from the damped force response curve. This value is determined by fitting a line to the peak-to-peak values of the damped force response curve. The fitted line is assumed to have a solution in the form,

$$
F(t)=F_{o} e^{-\zeta_{F} \omega_{d} t}
$$

$\mathrm{F}_{\mathrm{o}}$ represents the initial force at time $\mathrm{t}=0 \mathrm{~s}$. Obtaining this equation yields a value for $\zeta_{\mathrm{F}}$. Converting the value of $\zeta_{\mathrm{F}}$ to a percentile indicates the percentage of damping present in the system.

Simulation models which are subjected to high damping must be analyzed using a complex approach to handle the insufficient amount of numerical data during the damped period.

\footnotetext{
'During testing, the tanks are excited about a single axis through a pre-defined forcing function. The pre-defined force is then removed and the tank is allowed to dampen with no external, applied force.
} 


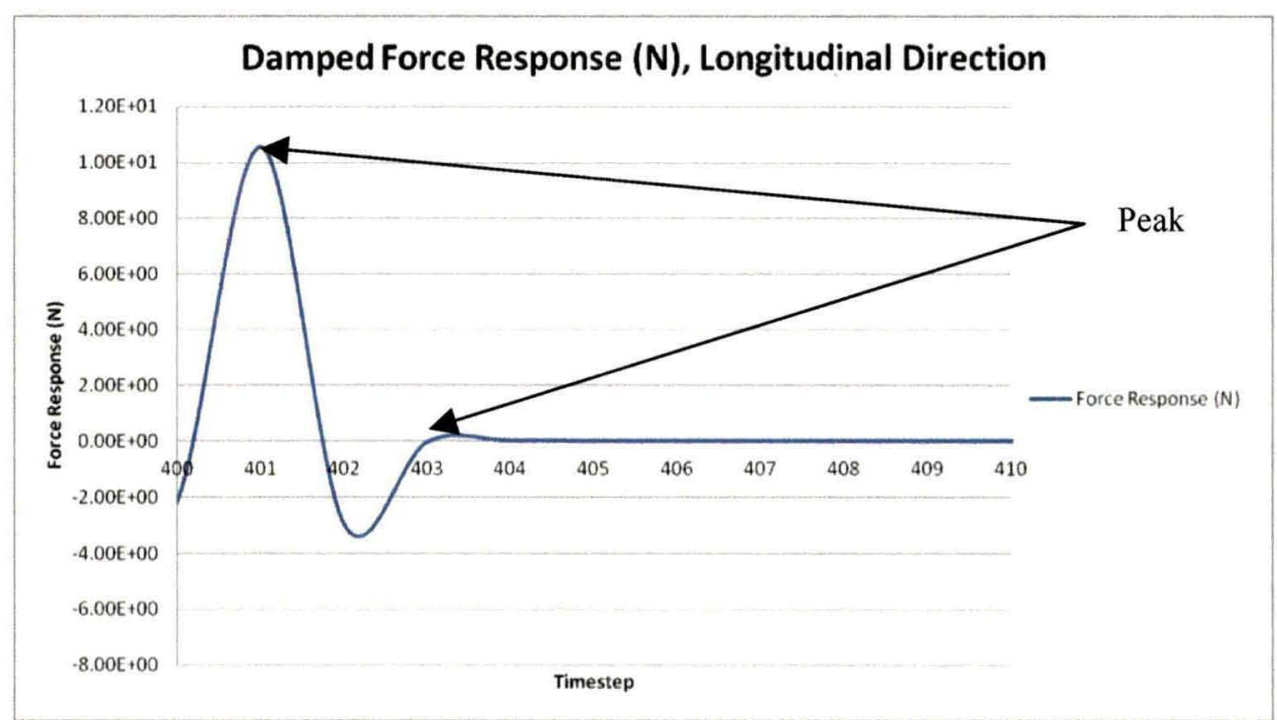

Figure 2. Damped Force Response Output, High Damping

Figure 2 illustrates the damping curve for a model with a large amount of damping. A simple, customary approach to analyzing damped systems is to exponentially curve-fit a line through the peak values of the oscillations. However, since there are only two peaks in Figure 2 worth considering for this approach, the curve-fit loses accuracy and the equation of the line is not valid. Therefore, a more accurate approach is needed for a model with a large amount of damping.

In keeping with the research objective of considering solutions to MDOF systems, the behavior of a large viscously damped system follows Newtonian Law and results in a similar equation to Eq. (1).

$$
M \ddot{u}+C \dot{u}+K u=0
$$

The viscously damped system under consideration is assumed to be $n$-DOF although for the purposes of this research investigation, $n=2 . M, C$ and $K$ are $n \times n$ square matrices. A general solution to Eq. (6) is in the form,

$$
u(t)=\Psi e^{\alpha t}
$$

where $\alpha$ is a complex number. $\Psi$ are associated vectors satisfying Eq. (8). Substituting Eq. (7) into Eq. (6) yields the characteristic equation, Eq. (8), to an $n$-DOF viscously damped system with no external forces acting on the system.

$$
\left(\alpha^{2} M+\alpha C+K\right) \Psi=f_{\alpha} \Psi=0
$$

Knowing that $f_{\alpha}$ is an $n \times n$ matrix, it is possible to obtain a non-trivial solution to the system of homogenous equations if the determinant of the system is zero, i.e.:

$$
\operatorname{det}\left(f_{\alpha}\right)=0
$$

The roots of the characteristic polynomial can take on three forms:

1. Real and Negative $(\alpha<0)$

2. Purely Imaginary $(\alpha=+\backslash-i \omega)$

3. Complex Conjugate Pairs $\left(\alpha=\zeta \omega_{\mathrm{n}}+\downarrow-i \omega_{\mathrm{d}}\right)$ provided system matrices are symmetric

ii The following formulas for MDOF viscous damped systems are taken from lecture notes written by Dr. Luis San Andres from Texas A\&M University. ${ }^{3}$ 
Furthermore, if the real part of $\alpha>0$, then the system is considered unstable. Working through Eq. (8) and Eq. (9) (referencing the notes from Dr. San Andres), one can extract $2 n$ eigenvalues, $\lambda$, and associated eigenvectors, $\phi$. The eigenvectors are represented in a matrix, $\phi_{r}=\left[\begin{array}{l}\psi_{r}^{1} \\ \psi_{r}^{2}\end{array}\right]_{2 n \times 1}$, where $\Psi_{\mathrm{r}}$ is an $n \times 1$ vector satisfying,

$$
\left[\begin{array}{cc}
0 & I \\
-K^{-1} M & -K^{-1} C
\end{array}\right]\left[\begin{array}{l}
\psi_{r}^{1} \\
\psi_{r}^{2}
\end{array}\right]=\frac{1}{\alpha_{r}}\left[\begin{array}{l}
\psi_{r}^{1} \\
\psi_{r}^{2}
\end{array}\right]
$$

Equation (10) is in a recognizable form to solve an eigenvalue problem. The $M, C$ and $K$ matrices are defined from the data outputs of the force vs. time and moment vs. time plots of the computational solutions. Substituting the $M, C$ and $K$ matrices into Eq. (10), the eigenvalue problem can be solved. The eigenvalues obtained from Eq. (10) essentially do not represent anything in terms of signal processing. They must be set equal to $1 / \alpha_{\mathrm{r}}$.

$$
\lambda=\frac{1}{\alpha_{r}}
$$

Because the behavior of the damping is underdamped, it is expected that the roots, $\alpha$, of the characteristic polynomial in Eq. (8) are to be complex conjugate pairs ${ }^{\mathrm{iii}}$ in the form,

$$
\alpha=\zeta \omega_{n} \pm i \omega_{d}
$$

Furthermore, if $\operatorname{Re}(\alpha)>0$, the system is considered to be unstable. Knowing the value of $\alpha$ allows the undamped and damped natural frequencies to be determined. The remaining damping parameter needed is the damping ratio, $\zeta_{\mathrm{F}}$, of the damped force response curve. Much like the low-damped systems, the damped force response curve is fitted with a line which is assumed to have an equation following Eq. (5). The value of $\zeta_{\mathrm{F}}$ is determined from the equation and converted to a percentile to indicate the amount of damping in the system.

\section{Free-Surface Tank}

\section{A. Set-up}

The intent of this research is to investigate the damping effects of different diaphragm shapes and materials implemented within spherical spacecraft propellant tanks. As with any comparative research, the investigation requires a control model to be used as a basis for drawing comparisons and formulating conclusions between all test models. The most appropriate type of model is a bare fuel tank known as the free-surface tank.

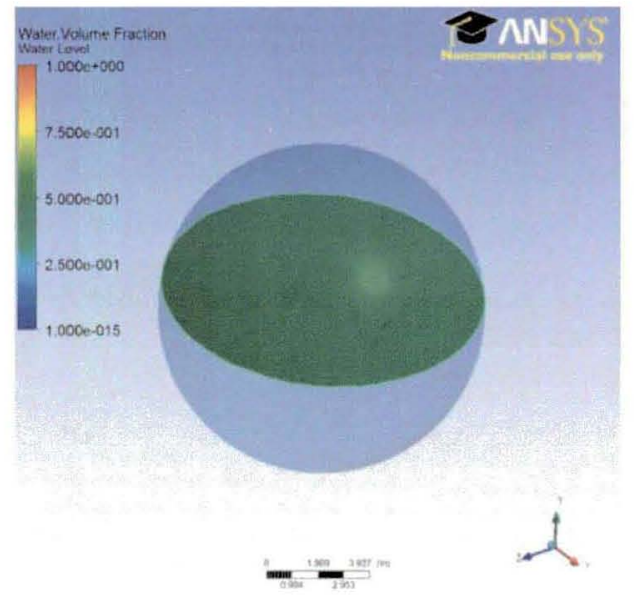

Figure 3. Free-Surface Tank ${ }^{\text {iv }}$

The free-surface tank, illustrated in Figure 3, is a propellant tank which is not implemented with a diaphragm or a baffle. Should the propellant within the tank become excited, there is no device within the tank to

iii Provided the matrices are symmetric.

${ }^{\text {iv }}$ The green iso-surface represents the water-level in the tank. 
induce damping. As such, the fluid is allowed to dampen naturally due to its viscosity. Viscous damping is considered to be the control for this research investigation. It is expected that the damping due to viscosity will be relatively low as compared to the other diaphragm-implemented tanks in this research. Therefore, the free-surface tank establishes a good baseline model with which to compare other diaphragm-implemented tanks and discern the impact the diaphragms have on damping.

The computational model's set-up begins by creating a CAD model in CATIA ${ }^{4}$, a computer-aided design software. The free-surface model is designed to have the same dimensions as the other spherical tanks in this research investigation. The inner diameter of the sphere is measured to be 12.549 ". For simplicity during computational analysis, the tank is designed without a thickness. Essentially, the CAD model is a non-hollow sphere with diameter 12.549". Once the complete model is created in CATIA, it is meshed using Pointwise ${ }^{6}$, a meshing software, to prepare it for ANSYS CFX ${ }^{5}$.

Pointwise creates a fine mesh over the surface of the model and defines the block (the mesh within the tank) to be a fluid. The fine mesh allows accurate results to be.achieved in the computational software. The side walls of the tank are rigid and thus are labeled as such in Pointwise. Defining the boundary as a wall and the block as a fluid allows the tank to be created without thickness as aforementioned.

\section{B. Computational Analysis}

Once meshed, the Pointwise grid file is imported into ANSYS CFX and the CFD simulation is defined. The simulation for the free-surface tank is designed as close as possible to the other tank models to ensure an accurate comparison can be made. The free-surface simulation is defined as a transient analysis as opposed to steady state analysis because it is assumed that the behavior of the fluid is time-dependent and thus the partial derivatives with respect to time, $\partial / \partial t$, are considered a necessary part of the solution. The simulation is designed with a sufficient total time to allow the solution to build and yield useful results. An appropriate timestep for the simulation must be chosen to avoid divergence within the residuals of the solution.

The free-surface tank is filled to $60 \%$ capacity. Past research has proven that the $60 \%$ fill-level of spherical propellant tanks results in the largest reaction forces of the sloshing liquid on the sidewalls of the tank ${ }^{7}$. Because the free-surface tank is not implemented with a diaphragm, there is a large free-surface of liquid within the tank which is in contact with the pressurant (in this case, the air within the tank). During excitation, there are large amounts of surface slosh within the tank and as a result, the computational data within the cells of the tank changes rapidly. To avoid a diverged solution, a timestep of $10^{-3}(\mathrm{~s})$ is chosen.

Solutions for each timestep are achieved when the iterative residuals are minimized below a value of $10^{-4}$. However, no more than 30 iterations (coefficient loops) are to occur per timestep as it is expected that the residuals will reach their criteria value $\left(10^{-4}\right)$ before 30 iterations. The computational scheme CFX uses to obtain a solution for the given simulations is a higher order backward Euler scheme.

During the simulation, monitors are placed at the boundaries (sidewalls) of the tank to monitor the reaction forces from the sloshing fluid within the tank. The oscillatory data collected from the force response is used to determine the natural frequency of the system. The model is excited by implementing a user-defined expression to displace the tank a set distance on either side of a neutral position at a specified frequency. The free-surface simulation is excited for 10 seconds before the fluid is allowed to dampen for an additional 8 seconds. To simplify the simulation, the effects of turbulence are neglected. Additionally, fluid buoyancy is considered which allows gravity to factor into the solution.

\section{Metallic Diaphragms}

\section{A. Set-up}

Standard practice of initial spacecraft design focuses on the need of a propellant management device within the propellant tank to dampen fuel slosh. Propellant tanks are commonly outfitted with flexible, rubber-like diaphragms which couple to the surface of the propellant and deform as the liquid sloshes. However, as of recent, the focus of propellant tanks during preliminary spacecraft design has been on the feasibility of metallic diaphragms. The premise of their function is to behave similar to flexible diaphragms in so much as they absorb the kinetic energy from fuel slosh and mitigate the adverse forces and moments the sidewalls of the propellant tank experience. Much like flexible diaphragms, metallic diaphragms deform as the propellant does and take on particular shapes at specific fill-levels. It is believed that at the critical $60 \%$ fill-level, the metallic diaphragm will form either a cratershape or a mountain-shape. ${ }^{8}$ 


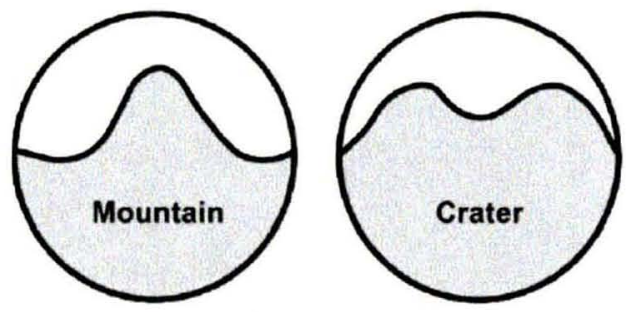

Figure 4. Mountain and Crater-Shaped Diaphragms ${ }^{9}$

A key parameter for diaphragm shape is the dimensions of a completely expanded diaphragm. There is no physical metallic diaphragm model for this research and thus, all metallic diaphragm dimensions reflect those of a flexible diaphragm provided by ATK/PSI ${ }^{\mathrm{v}}$. Using the same dimensions across both metallic and flexible models allows more validity in the comparative analysis between the two types of diaphragms. The dimensions of the fullyexpanded flexible diaphragm (and by default, the metallic diaphragm) are outlined in Table 1.

Table 1. Dimensions and Constants of ATK/PSI Diaphragm

\begin{tabular}{|c|c|}
\hline Diaphragm Dimensions and Constants $^{\mathrm{vi}}$ & Value \\
\hline Sample Thickness (in) & 0.063 \\
\hline Diameter (in) & 12.12 \\
\hline Volume $\left(\mathrm{in}^{3}\right)$ & 466.097 \\
\hline Surface Area $\left(\mathrm{in}^{2}\right)$ & 230.741 \\
\hline${ }^{\mathrm{vii}}$ Density of Sample $\left(\mathrm{kg} / \mathrm{m}^{3}\right)$ & 559.709 \\
\hline
\end{tabular}

While modeling either diaphragm, two key parameters must be satisfied from Table 1: the volume and the surface area. The volume of the various diaphragm-shape models designed in CATIA must correspond to the volume of the diaphragm-shape at the respective fill level. Additionally, the surface area of the diaphragm at any fill-level must always remain constant due to conservation of material.

The process by which a model is created in CATIA is complex, requiring that the clamping hemisphere (the hemisphere to which the diaphragm is attached) and the diaphragm be made as separate entities to validate dimensions of the respective parts. The clamping hemisphere has an inner diameter of 12.549 " and thus a volume of $517.364 \mathrm{in}^{3}$. The equations below illustrate how a diaphragm shape volume corresponding to a $60 \%$ fill-level is determined.

$$
\begin{gathered}
V_{\text {Clamping Hemisphere }}+V_{\text {Diaphragm }}=V_{\text {Total }} \\
V_{\text {Total }} * 0.60=V_{60 \%} \\
V_{60 \%}-V_{\text {Clamping Hemisphere }}=V_{\text {Diaphragm } 60 \%}
\end{gathered}
$$

Using optimization techniques in CATIA, a crater-shape diaphragm and a mountain-shape diaphragm are modeled. The clamping hemisphere, together with the respective diaphragm shape, achieves a $60 \%$ fill-level volume of $590.076 \mathrm{in}^{3}$ with the diaphragm surface area equaling $230.741 \mathrm{in}^{2}$, the target value. It is important to note that the complete model is one solid shape and does not incorporate thicknesses into the individual pieces. Creating the complete model as one solid model simplifies the meshing approach and allows for a proper computational analysis to be performed.

\footnotetext{
${ }^{\mathrm{v}}$ The flexible diaphragm provided by ATK/PSI is used for experimental research at the Embry-Riddle Aeronautical University Fuel Slosh Research Facility.

${ }^{v i}$ Dimensions of diaphragm are taken when diaphragm is fully expanded.

${ }^{v i i}$ Density of sample is derived from a previous test. Density is only applicable to flexible diaphragm.
} 
The complete models are imported into Pointwise to be meshed. Pointwise allows a single fluid domain to be defined within the complete diaphragm/hemisphere model. Additionally, Pointwise creates a fine mesh over and within the CATIA models. It is important that a fine mesh is created so as to achieve a well-defined block which allows for more accurate results. Only one block is created and labeled as a fluid. The metallic diaphragm, due to its rigid-like properties, is defined as a wall since no deflection/deformation is expected during simulations.

\section{B. Computational Analysis}

Once meshed, the grid file is imported into ANSYS CFX and the CFD simulation is defined. The simulations for the metallic diaphragm crater-shape and mountain-shape are set-up identical to allow an appropriate comparison to be made against each other and other models in this research. Like the free-surface model, the metallic diaphragm models are defined as transient analysis.

The metallic diaphragms are modeled as representing a specific point in time in which the metallic diaphragm has depleted to a fill-level representing the $60 \%$ fill-level. It is assumed that there is minimal to no pressurant within the tank during this simulation and thus, there is no free-surface within the tank. Because there is no free-surface, there will be no surface slosh. The internal reaction forces on the sidewalls of the tank will arise from sub-surface vortices and currents within the liquid. As a result, the data within each computational cell of the tank will not be changing as fast as it was in the free-surface test. Thus a larger timestep of $10^{-2}$ can be chosen without risking divergence.

The remainder of the computational set-up for the metallic diaphragms is nearly identical to the set-up for the free-surface simulation in regards to timesteps, computational scheme, force monitors and buoyancy. However, the most important parameter which distinguishes the metallic diaphragm simulations from the flexible diaphragm simulations is the elimination of the need for fluid-structure interaction (FSI). The rigid nature of the metallic diaphragms does not allow them to couple to the surface of the liquid. Though the diaphragms will deplete as the propellant depletes, the surfaces of the diaphragm and propellant remain uncoupled. Essentially, the simulations are considering a propellant tank that is $100 \%$ full, with an alternative top hemisphere-shape (the diaphragm) which reduces the total "spherical" tank volume down to a " $60 \%$ fill-level". Since ANSYS CFX considers the diaphragm shape as a rigid wall, and not a diaphragm, there is no FSI to incorporate which greatly simplifies the simulation and cuts down on simulation time.

\section{Flexible Diaphragms}

\section{A. Set-up}

The set-up for modeling the flexible diaphragms is significantly different from that of the metallic set-up due to the requirements of the computational solver used to model the FSI between the diaphragm and the propellant. Unlike the metallic diaphragm models which are created as one solid model with the diaphragm shape forming the top of the propellant hemisphere, the diaphragm for the flexible diaphragm models must be created as a 3-D part and fixed to the other parts. Allowing the diaphragm to be its own entity allows the computational solver to consider the diaphragm for both the fluid and structures solution.

There are three parts which comprise the flexible diaphragm complete model: the diaphragm, the propellant and the pressurant.

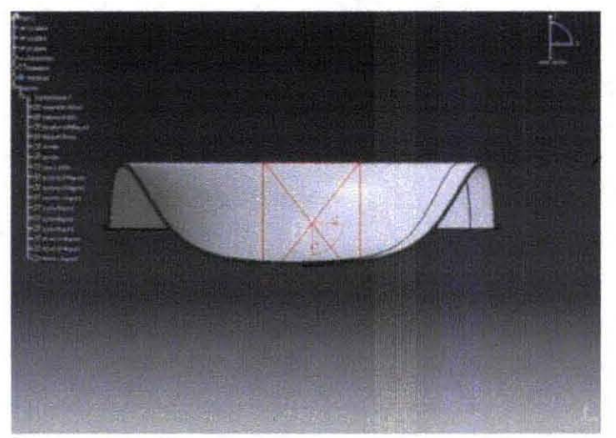

Figure 5. Cross-Sectional View Diaphragm 60\%

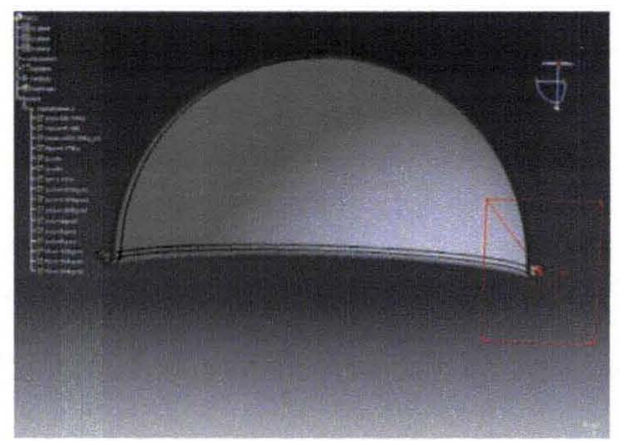

Figure 6. Cross-Sectional View Diaphragm $100 \%$ 


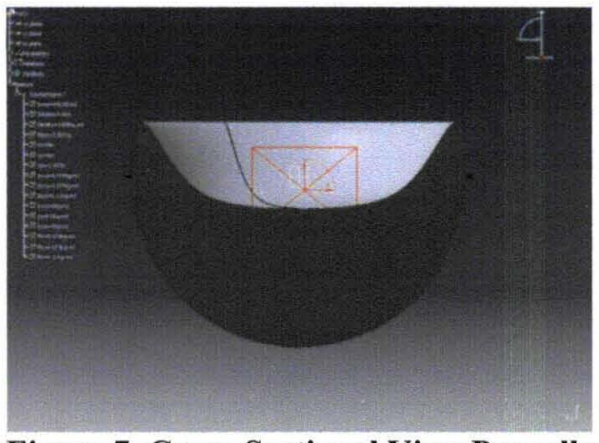

Figure 7. Cross-Sectional View Propellant $60 \%$

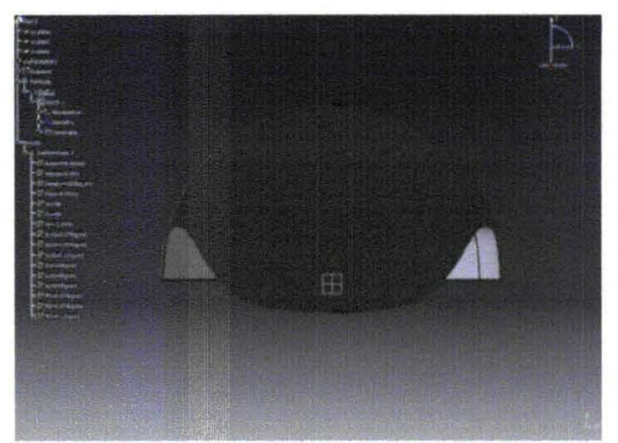

Figure 8. Cross-Sectional View Pressurant 60\%

The first of the three parts, the diaphragm, is further broken down into a diaphragm shape corresponding to a $60 \%$ fill-level and a shape corresponding to a $100 \%$ fill-level. The $60 \%$ fill-level diaphragm (a crater-shaped diaphragm), illustrated in Figure 5, is used for current research. The $100 \%$ fill-level diaphragm, is used to test propellant depletion scenarios in simulations which will occur later in the research. The second part, being called the propellant, refers to the hemisphere that contains the propellant, illustrated in Figure 7. The propellant is modeled in CATIA as a solid part for computational purposes. The third part, the pressurant, refers to the hemisphere which contains the pressurant, illustrated in Figure 8. The pressurant is also modeled as a solid part in CATIA for computational purposes. The complete tank set-up for the current research fixes the $60 \%$ crater-shaped diaphragm to the propellant and pressurant respectively.

\section{B. Computational Analysis}

The computational set-up for the flexible diaphragm simulations is significantly different from the computational set-up for the metallic diaphragms due to the consideration of FSI. Because there is fluid-structure interaction taking place in the flexible diaphragm simulations, ANSYS Workbench must be used to determine a solution to the simulation. ANSYS Workbench allows the solver to calculate a two-way coupled solution between ANSYS CFX, the fluids solver, and ANSYS Transient Structural, the structural solver.

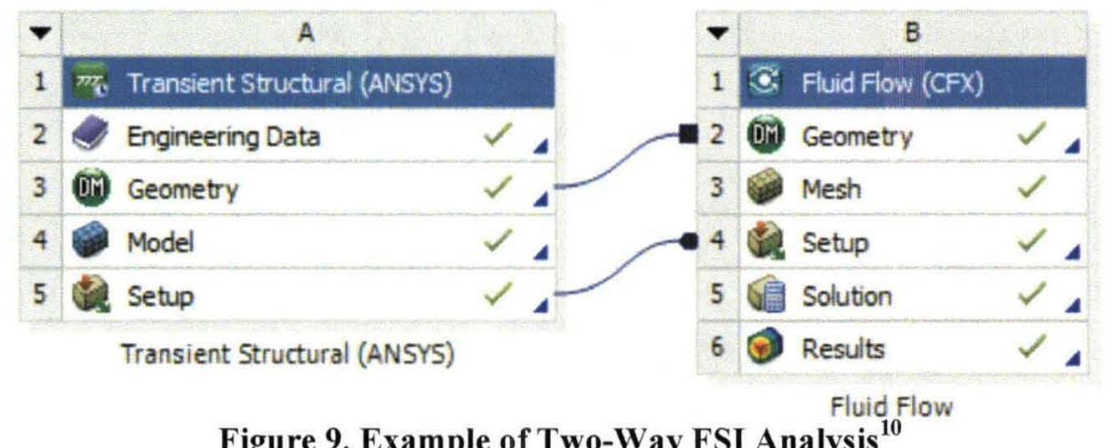

Figure 9 illustrates how the two-way coupled solution is set-up with the structural solver and the fluids solver; each sharing the geometry of the model and the set-up files necessary to define boundaries and initial conditions of the model.

During the set-up and definition of the simulation, ANSYS Multi-field with physics is chosen as the external solver coupling option. This allows the structural input files to be passed from the structures solver into the fluids solver, CFX, to determine the FSI. Since all timesteps are coupled in a transient multi-field analysis, the solver solves the structural field and uses that solution to obtain a solution to the fluids field, thus creating a two-way coupled solution within the same iterative timestep.

The timestep chosen for the FSI simulation is critical in obtaining a solution within a reasonable amount of simulation time. The meshed tank and diaphragm is a very complex set-up with a fine grid containing many cells. Not only is the fluid displacing, the flexible diaphragm is displacing, adding to the computational solver complexity. The structures mesh and the fluids mesh are created together in ANSYS mechanical to ensure the cells along the boundaries of the individual domains are node-matched. Having node-matched cells on the boundaries ensures the induced deformations on the boundaries do not result in high skew angles of the cells or mesh folding, two conditions which will cause the solver to crash. Additionally, it is assumed that the largest deformations occur at the 
middle of the diaphragm. Thus, the mesh stiffness is increased around the edges of the diaphragm to prevent unrealistic deformations at areas of the diaphragm which are not expected to deform. However, because of the large deformations expected at the center of the diaphragm, a timestep of $10^{-4}$ is chosen to avoid divergence.

Much like the set-up for the metallic diaphragms, the tank excitation is defined through a user-defined function and is allowed to translate a set distance on either side of a pre-defined neutral position. Other parameters such as buoyancy and turbulence are also defined much like the metallic diaphragm set-up.

Similar to the metallic diaphragm set-up, coupled solutions for each timestep are achieved when the iterative residuals are minimized below a criteria value of $10^{-4}$. However, no more than 10 iterations (coefficient loops) are to occur per timestep as it is expected that the residuals will reach their criteria value $\left(10^{-4}\right)$ before 10 iterations. As previously stated, the coupled solution sequence solves the all structural fields before the CFX fields for each iteration. The fluids solver then uses higher order backward Euler equations to obtain a solution for the transient scheme, thus achieving a coupled solution.

\section{Results}

\section{A. Free-Surface Tank}

Past research has been conducted on free-surface spherical propellant tanks at the Embry-Riddle Fuel Slosh Test Facility. The results from that research yielded a natural frequency value of the spherical propellant tank system which is useful as a preliminary value for the current research investigation. Aside from the computational side of the research, there is an experimental side of the investigation which impacts various parameters in the computational set-up of the various simulations. ${ }^{\text {vii }}$ The natural frequency value of the free-surface tank obtained from past experimental tests, $\sim 1.78 \mathrm{~Hz}$, is unable to be used for computational purposes due to limitations in the experimental set-up. Therefore, the preliminary computational simulations of the current free-surface tank consider an excitation frequency of $1.7321 \mathrm{~Hz}$. The preliminary excitation frequency value is not expected to be the exact undamped natural frequency of the system but it is an acceptable starting frequency useful for determining the first mode natural frequency of the system.

Results of the free-surface simulation are obtained upon the simulation completing normally. CFX-Solver, the CFD solver used by ANSYS to compute the solution to the free-surface simulation, outputs various plots including force response vs. time and moment response vs. time. The largest reaction loads the tank experiences occur as a result of the forces, specifically, the force response in the direction of excitation, the longitudinal direction.

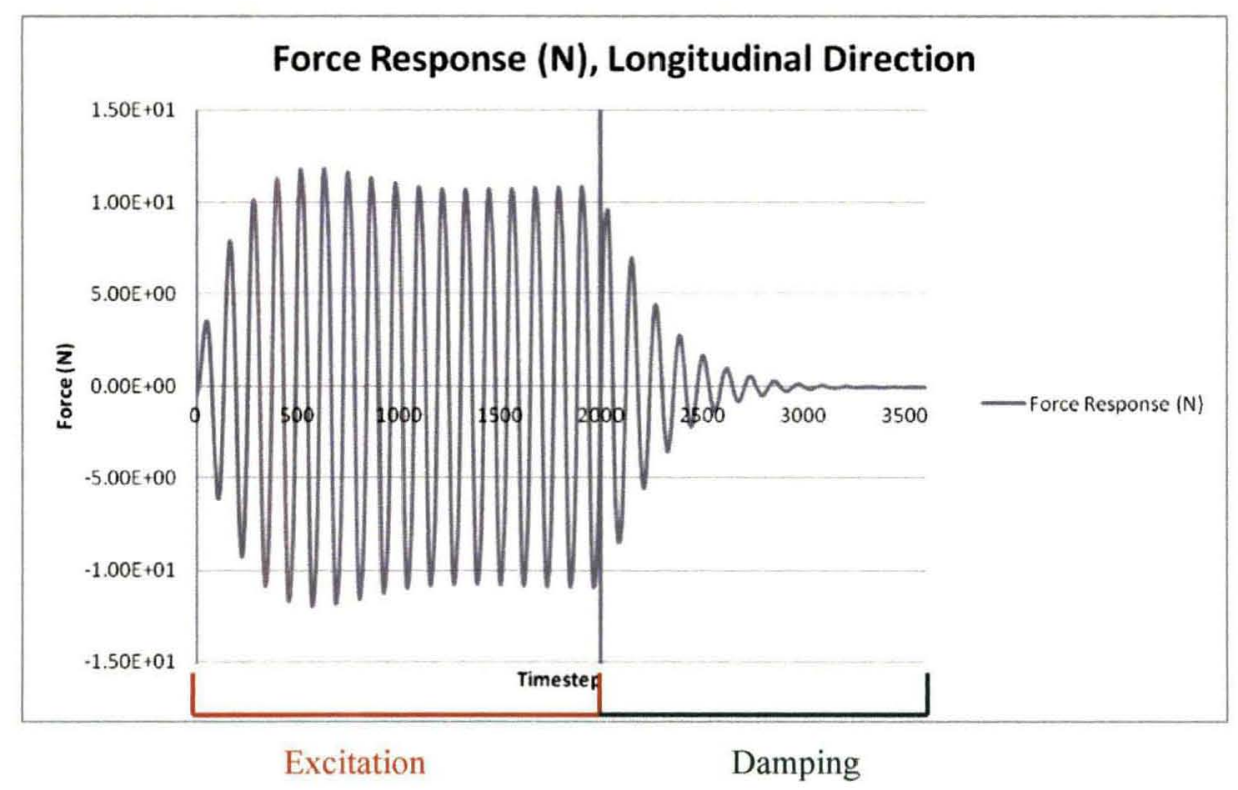

Figure 10. Force Response Output, Computational Free-Surface Simulation

viii Experimental simulations are aimed at replicating computational simulations for comparison purposes. However, limitations in the experimental equipment impact certain computational set-up parameters. 
Figure 10 is the output plot obtained from CFX-Solver for the initial simulation of the spherical freesurface tank. The plot represents the internal, longitudinal reaction forces the tank experiences as a function of the simulation timestep during the excitation and the damping of the simulation. During excitation, the tank is subjected to a constant forcing function defined by the excitation frequency of the system. At the $2000^{\text {th }}$ timestep, the forcing function ceases, the tank stops displacing and the fluid is allowed to dampen within the tank. Because this is a freesurface simulation, the only damping taking place is viscous damping.

Liquid hydrazine, a common spacecraft propellant, has approximately the same density and viscosity as water. ${ }^{11}$ Therefore, the material properties of water are used to define the fluid in this and all other simulations in this research. Water is not the most viscous of liquids so it is expected that the viscous damping taking place in the tank is minimal, especially without the presence of a diaphragm or any type of PMD. This assertion is confirmed by referencing the damping period in Figure 10. The damping period is highly underdamped with the reaction forces oscillating between $\pm 11 N$ and gradually reducing to zero.

Once it is confirmed that the longitudinal reaction forces behave as initially expected, the data from this output are imported into a user-defined MATLAB algorithm to determine the natural frequencies of the system. This research explores various types of signal decomposition in order to determine the natural frequencies and damping ratios of the force response plots. The main factor affecting the choice of signal analysis is that of the damping data. A highly underdamped force response, such as the free-surface tank, has a significant amount of data, well-defined oscillation peaks and a well-defined damping curve. With these conditions, this research is able to implement spectral analysis to determine the natural frequencies and damping ratio of the system through this force response.

The spectral analysis utilized in this research considers a two-fold approach. First, the force response data is input into a user-defined MATLAB algorithm to determine the undamped and damped natural frequencies of the system through a Fast Fourier Transform (FFT). The data is divided into excitation data and damping data to determine the undamped and damped natural frequencies respectively. This approach is taken so the excitation data does not influence the damped natural frequency and the damping data does not influence the undamped natural frequency. The second part of the spectral analysis implements the force response data into a second MATLAB algorithm to create a 2-D spectrogram. Again, the data is divided into excitation data and damped data so neither data influences the natural frequency results of the other. The spectrogram is used to determine the undamped and damped natural frequencies of the system. The results are compared to the natural frequencies obtained through FFT to ensure the values being reported are accurate.
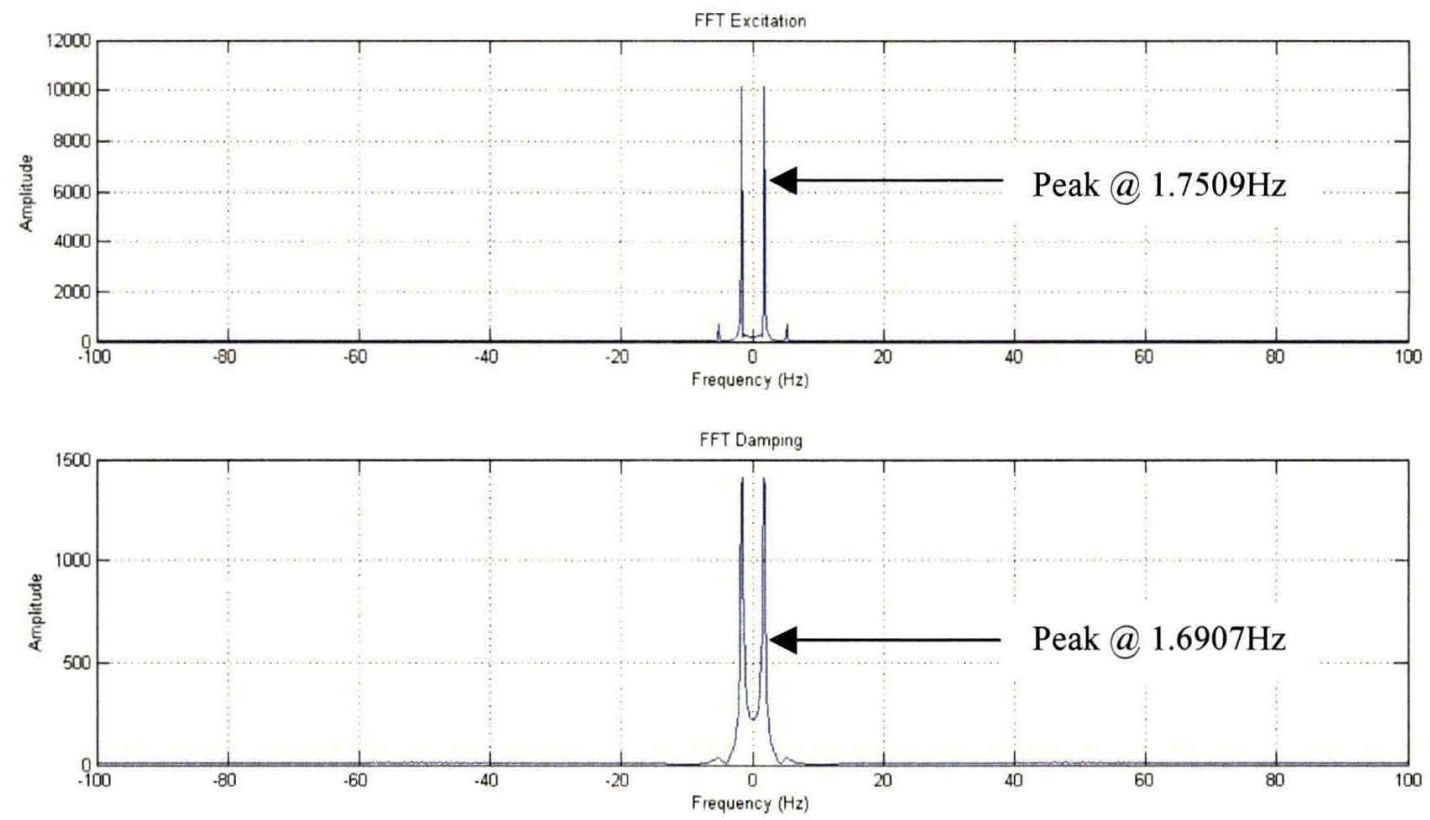

Figure 11. Fast Fourier Transform Plot of Free-Surface Force Response, Longitudinal Direction

Figure 11 represents the FFT plots the MATLAB algorithm generates based on the force response data from Figure 11. The top plot represents the FFT of the excitation data only. Applying a shift to the plot to centers it 
about $0 \mathrm{~Hz}$. For realistic applications and in keeping with the focus of this study, only the peaks occurring in the positive frequency spectrum are considered. The positive peak in the top plot is located at $1.7509 \mathrm{~Hz}$ which represents the undamped natural frequency of the system.

The bottom plot represents the FFT of the damped data only. Again, applying a shift to the original plot centers it about $0 \mathrm{~Hz}$ as depicted in Figure 11. Considering only the positive peak for the FFT damped data, the peak is located at $1.6907 \mathrm{~Hz}$, the damped natural frequency of the system.

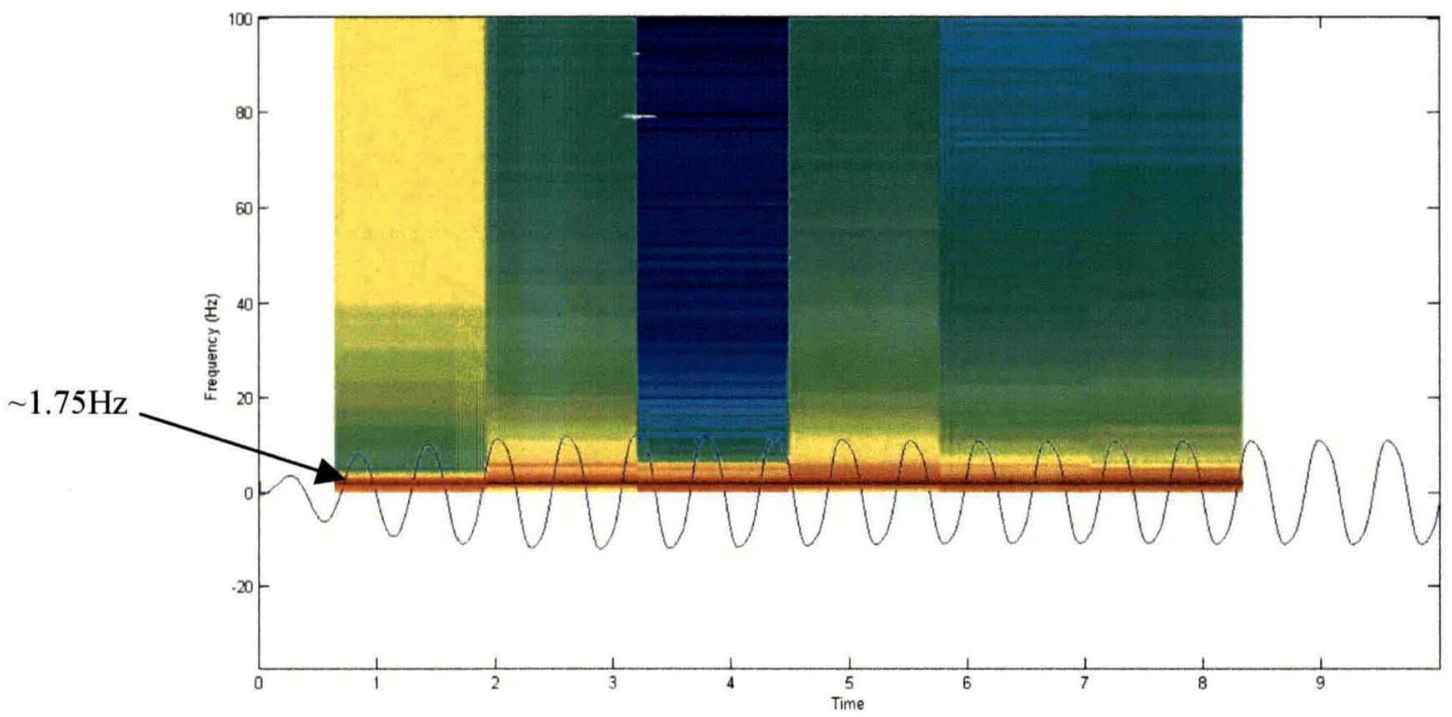

Figure 42. 2-D Spectrogram of Free-Surface Excitation Force Response, Longitudinal Direction

Spectral analysis using FFT plots is a very accurate approach to determine the undamped and damped natural frequencies of the force response signal. However, the FFT plots fail to provide any timing information of the signal; information useful in determining how the behavior of the signal strength is varying with respect to time. Therefore, this research investigation formulates 2-D spectrograms to analyze the frequency of the signal with respect to time while considering the strength of the signal.

Figure 12 illustrates the 2-D spectrogram of the excitation force response data from the free-surface tank. In addition to the spectrogram, the excitation force response curve is also plotted in the same figure. The principle axis of this spectrogram has the frequency on the vertical axis and the time on the horizontal axis. The color of the spectrogram represents the strength of the signal with blue indicating a weak signal and red indicating a strong signal. The red horizontal line at the bottom of the spectrogram corresponds to a strong signal at approximately $1.75 \mathrm{~Hz}$. Referencing the FFT of the excitation data in Figure 14, it is noted that the undamped natural frequency of the free-surface simulation is $1.7509 \mathrm{~Hz}$. The spectrogram of the excitation force response data is accurate in verifying the undamped natural frequency obtained through FFT of the free-surface simulation. It is also important to note that the horizontal line is a constant red color throughout the spectrogram. This is to be expected as the constant red line represents the strength of the undamped natural frequency of a harmonic signal with a constant period and amplitude. 


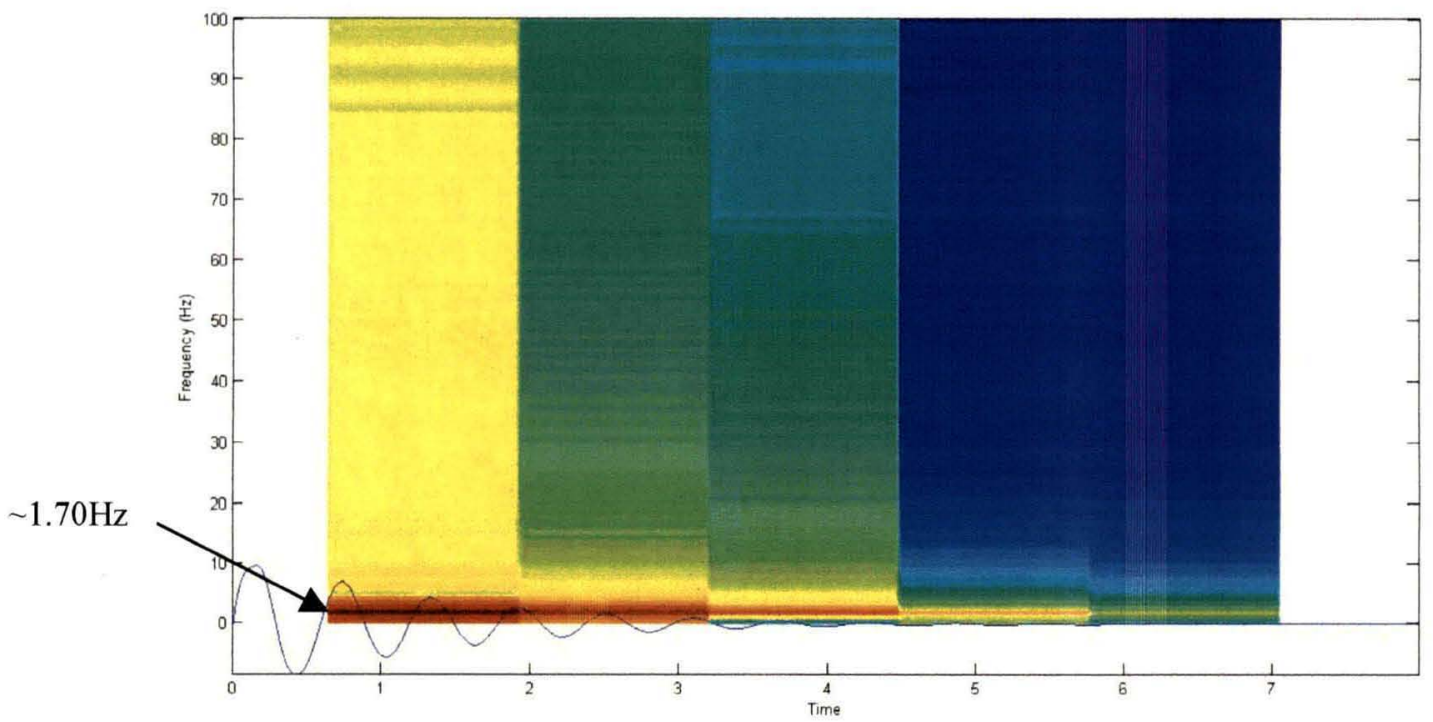

Figure 13. 2-D Spectrogram of Free-Surface Damped Force Response, Longitudinal Direction

Once the spectrogram for the excitation data has been formulated and the results confirmed, a spectrogram of the damping data is formulated. Figure 13 illustrates the 2-D spectrogram of the damped force response data from the free-surface tank. In addition to the spectrogram, the damped force response curve is also plotted in the same figure. The axis and color references are applied the same way as in Figure 12. The red horizontal line at the bottom of the spectrogram corresponds to a strong signal at approximately $1.70 \mathrm{~Hz}$. Referencing the FFT of the damped data in Figure 14, it is noted that the damped natural frequency of the free-surface simulation is $1.6907 \mathrm{~Hz}$. The spectrogram of the damped force response data is accurate in verifying the damped natural frequency obtained through FFT of the free-surface simulation. It is also important to note that the horizontal line is progressively diminishing in red color as time progresses. As the signal decreases in strength and amplitude, the red line indicating the strength of the damped natural frequency decreases in strength in accordance with the signal.

The natural frequencies of the free-surface system are extremely important parameters for preliminary spacecraft design. In addition to the natural frequencies, the damping ratio of the force response curve, $\zeta_{\mathrm{F}}$, is another useful parameter for understanding the damping behavior of the fluid. The damping ratio is simply found by curvefitting the peak-to-peak points of the damped force response curve. The solution to the curve-fit is assumed to be in the form of Eq. (5).

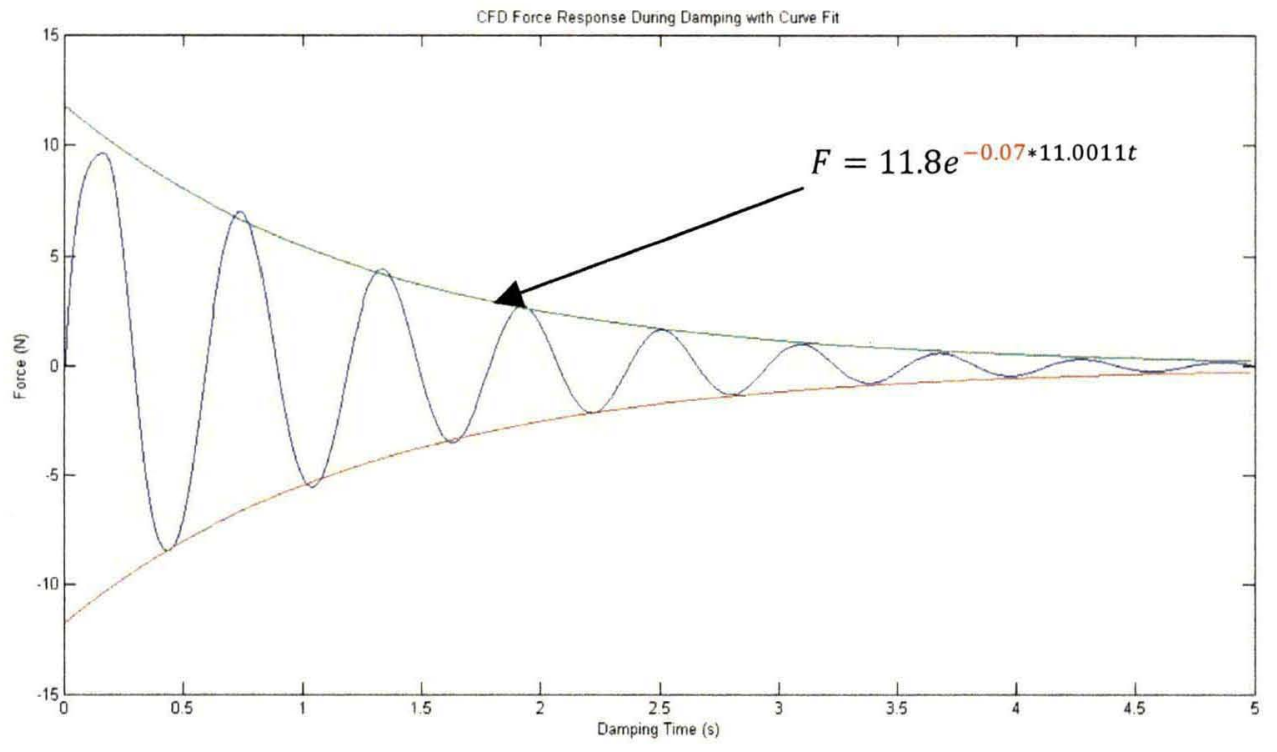

Figure 14. Free-Surface Damped Force Response with Curve-Fit, Longitudinal Direction 
Figure 14 illustrates the damped force response curve of the free-surface tank simulation with the curve-fit lines. Comparing the curve-fit equation in Figure 14 to Eq. (5), the damping ratio of the damped force response, $\zeta_{\mathrm{F}}$, is 0.07 or $7 \%$ damping. $7 \%$ damping is an acceptable damping value and is feasible for a free-surface simulation.

The second part to this research study investigates the behavior of the fluid during the simulation. Past research conducted at ERAU simply calculated the damping parameters of the free-surface tank and did not consider important physical details in the computational simulation results. Research on the free-surface tank is interested in how ANSYS CFX models the velocity vectors of the fluid and how accurate the surface of the fluid is modeled, focusing on behaviors such as sub-surface vortices. These fluid behaviors are important for further understanding the internal reaction forces on the tank and are good starting points for designing PMD's to counteract these potentially adverse behaviors.

ANSYS CFX-Post is the post-process module in the CFX software package that allows the results of the CFD simulation to be analyzed. Although there are many parameters and variables to potentially consider for analysis, only the fluid velocity vectors and the free-surface of the liquid are studied.

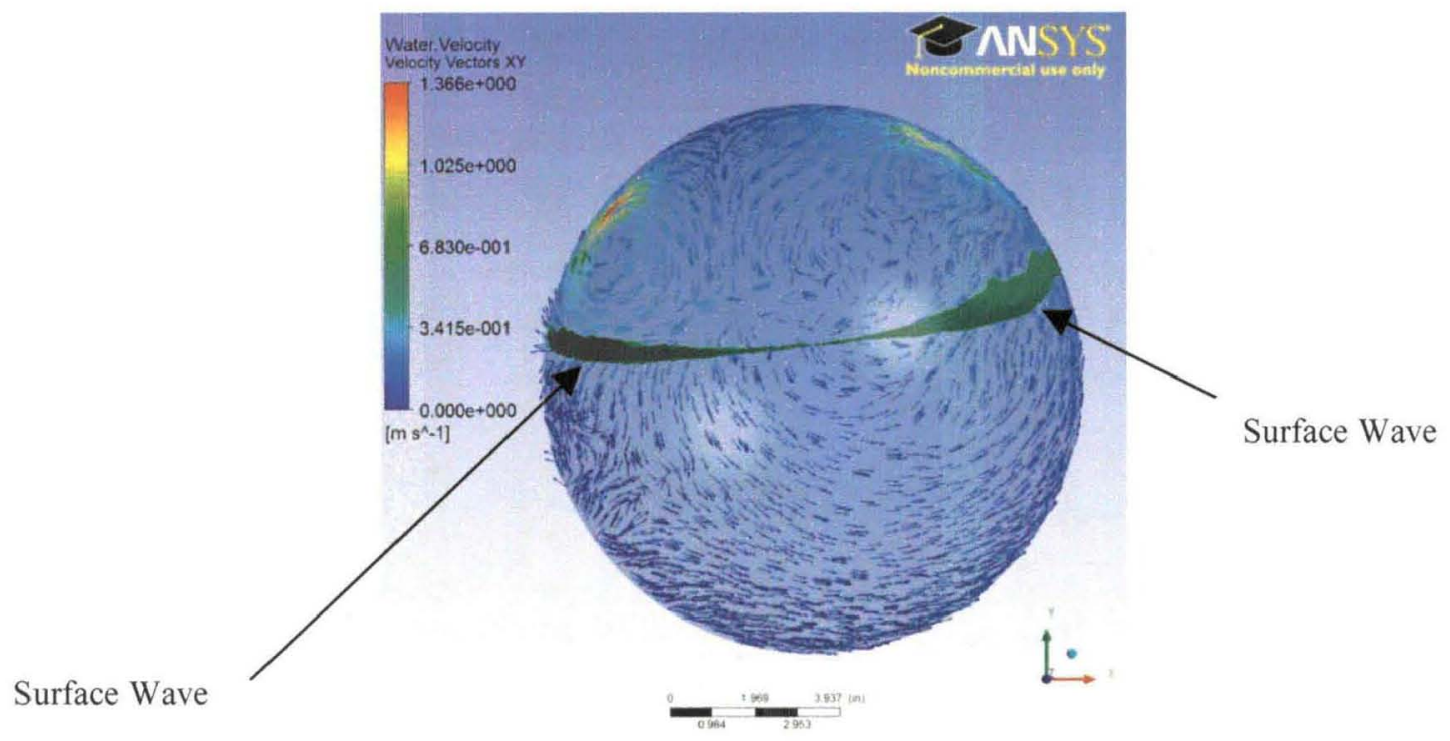

Figure 15. Velocity Vectors During Damping of Free-Surface Simulation, $t=10.08 \mathrm{~s}$

Figure 15 captures the velocity vectors of the fluid and the free-surface behavior during the damped portion of the simulation. Taken at time 10.08 seconds, the damping of the fluid within the tank has just initiated. Even though the motion of the tank has ceased, the liquid continues to slosh within the tank, displacing side-to-side. This is confirmed by examining the velocity vectors of the propellant. Examining the vectors on the left side of the tank, the majority of the vectors are oriented down and toward the middle of the tank. As such, the free-surface on this side of the tank is forced down so much so that a surface wave forms. Additionally, examining the right side of the tank, the propellant velocity vectors are oriented up and to the outside of the tank. As such, the vectors induce a surface wave within the tank. The surface waves formed during damping provide the internal forces on the sidewalls of the tank. As damping time progresses, the surface waves decrease in size and thus, decrease in strength. However, their presence within the tank is concerning due to the adverse effects they possess. Therefore, propellant tanks are implemented with diaphragms to combat the adverse forces and moments induced by the waves. The effects of the diaphragms are presented in the proceeding sections of this investigation.

\section{B. Metallic Diaphragms}

The use of metallic diaphragms in spacecraft propellant tanks is becoming a more accepted design consideration in the astronautical field as of recent. However, very little data exists as to the behavior of these diaphragms during common fuel slosh scenarios. From initial testing, it is believed that these diaphragms could work just as well, if not better than the current "rubber-like" flexible diaphragms. This research aims at further investigating the behaviors of the metallic diaphragms and exploring the comparisons to the flexible diaphragms. 


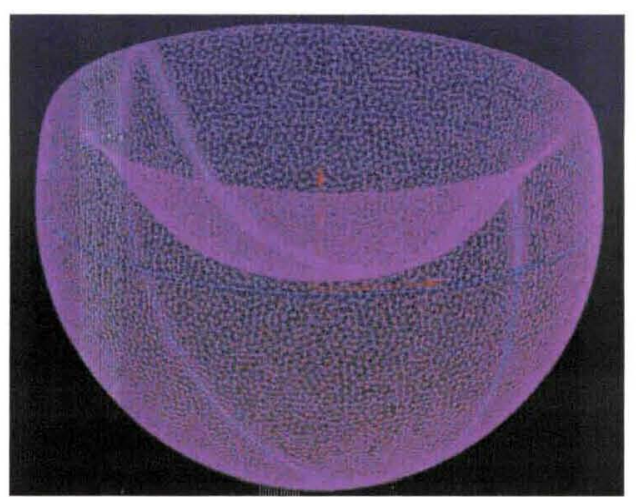

Figure 165. Meshed Tank with Rigid, Crater-Shaped Diaphragm

Figure 16 illustrates the propellant tank implemented with a rigid ${ }^{\mathrm{ix}}$, crater-shaped diaphragm. Computational testing on this tank begins using an excitation frequency of $2.2 \mathrm{~Hz}$ because that is the frequency which significantly excites the force responses within the tank during the frequency sweeps. It is not believed that this frequency is the natural frequency of the system but it should yield useful results for determining the first mode natural frequency of the system. Signal analysis using eigenvalues confirms the specific values of the undamped and damped natural frequencies found using this excitation frequency.

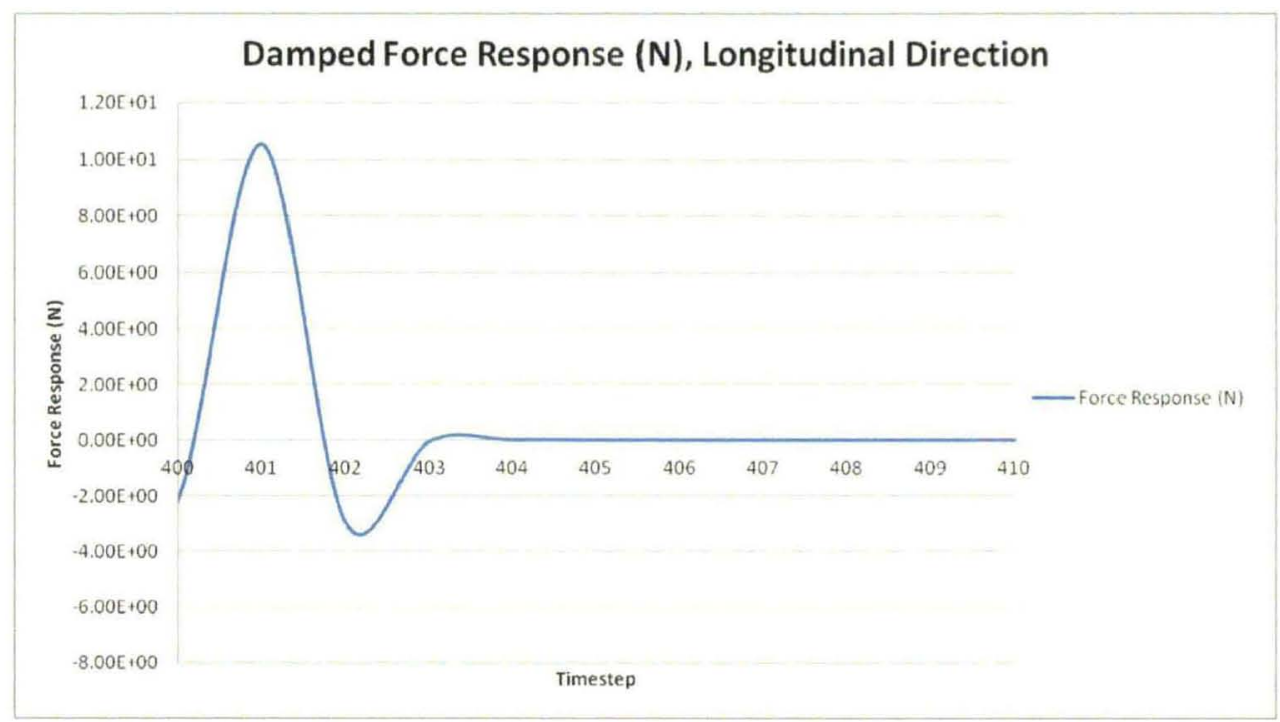

Figure 17. Rigid Crater-Shaped Diaphragm Damped Force Response, Longitudinal Direction

The simulation for the rigid, crater-shaped diaphragm tank is performed by exciting the tank for a predefined length of time and then stopping the motion, allowing the fluid within the tank to settle. Once the tank excitation ceases, the normal force response curve in the longitudinal direction follows Figure 17. Unlike the freesurface simulation, the rigid, crater-shaped diaphragm simulation dampens within four timesteps, or 0.2 seconds. This highly damped simulation is difficult to analyze because of the lack of data contained in four damped timesteps. Nonetheless, the data during damping is input into a user-defined MATLAB algorithm to solve the complex eigenvalue problem. The second degree of freedom used in the eigenvalue problem is the reaction moment generated from the reaction force in the longitudinal direction. This moment is the second largest internal load applied on the system and is thus the appropriate choice when adding data from a second degree of freedom. Furthermore, adding a second degree of freedom to the analysis improves the accuracy of the result. The results of the eigenvalue algorithm are displayed in Table 2.

\footnotetext{
ix The metallic diaphragms are commonly referred to in this research as "rigid diaphragms" to completely distinguish them from the "rubber-like" flexible diaphragms.
} 
Table 2. Rigid, Crater-Shaped Diaphragm Damping Results

\begin{tabular}{|c|c|}
\hline Parameter & Value \\
\hline$\omega_{\mathrm{n}}(\mathrm{rad} / \mathrm{s})$ & 8.6922 \\
\hline$\omega_{\mathrm{d}}(\mathrm{rad} / \mathrm{s})$ & 7.897 \\
\hline$\zeta$ & 0.4179 \\
\hline
\end{tabular}

Table 2 presents the damping results taken from the 2-DOF eigenvalue problem computed by MATLAB. MATLAB computes the natural frequencies of the system in $\mathrm{rad} / \mathrm{s}$ but converted to $\mathrm{Hz}$, the undamped natural frequency of the system is $1.3834 \mathrm{~Hz}$ and the damped natural frequency of the system is $1.2568 \mathrm{~Hz}$.

The free-surface simulation validated the accuracy of the calculated natural frequency values by plotting a spectrogram and comparing the results of the spectrogram to the results of the FFT. The data used in those calculations was considering a single degree of freedom. For the rigid, crater-shaped diaphragm simulation, the data imported into the eigenvalue problem are 2-DOF and thus, the results obtained represent a coupled solution. The process of creating a spectrogram from coupled data becomes exceedingly difficult and is outside the scope of the current research investigation. Methods of developing spectrograms from coupled data will be explored in future studies of this research. Although the process of determining the natural frequencies of a signal from eigenvalues is a very accurate method, the natural frequency data remains to be verified.

MATLAB also generates the damping ratio, $\zeta$, of this system from the coupled, damped force response data. This damping ratio represents the damping of the position vs. time curve of the mass of the system. Although this value is important to understanding the behavior of the system, it is not currently applicable to this part of the research. ${ }^{\mathrm{x}}$ This research is concerned with obtaining the damping ratio of the force response curve, $\zeta_{\mathrm{F}}$. After many attempts to curve-fit a peak-to-peak curve to the force response curve in Figure 17, it is determined that a curve-fit cannot be obtained due to an insufficient amount of data and lack of peaks. Therefore, the damping ratio of the force response curve cannot be found at this time.

Once the necessary damping parameters are obtained through the MDOF eigenvalue problem, force responses from additional fuel slosh scenarios are considered before analyzing the fluid behavior in the rigid, cratershaped diaphragm simulation. All simulations up to this point have been performed in a1G environment. This research investigates performing the exact same $1 \mathrm{G}$ simulation but inducing different gravitational effects on the tank to study how the fluid behaves. It is hypothesized that gravity will not affect the fluid behavior because the fluid dynamics are a function of system mass, not weight.

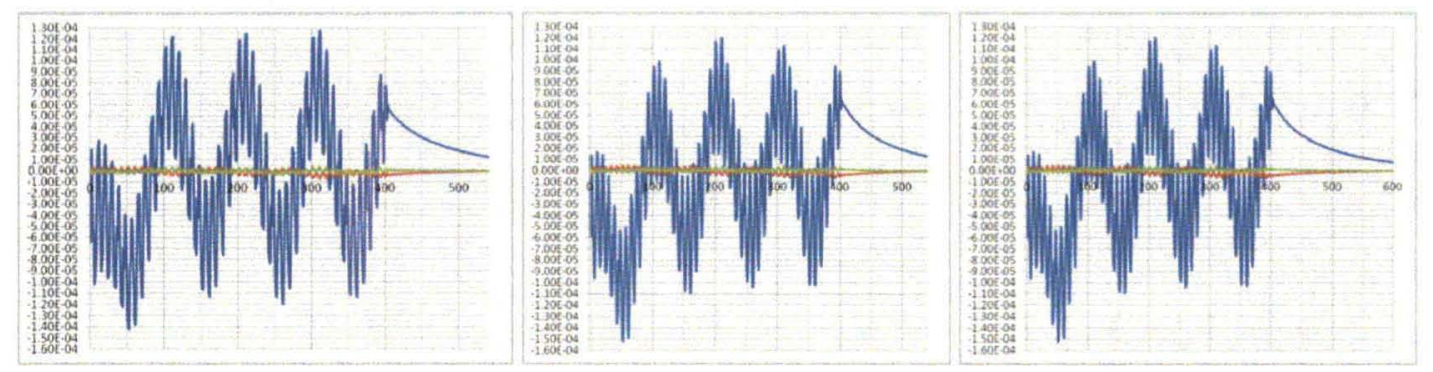

Figure 18. Rigid Crater-Shaped Diaphragm Tangential Force Response, 0G, 1G, 5G Respectively, Longitudinal Direction

Figure 18 compares the tangential force responses of the rigid, crater-shaped diaphragm tank simulated in $0 \mathrm{G}, 1 \mathrm{G}$ and $5 \mathrm{G}$ environments respectively. Comparing the three outputs, the tangential force responses are identical, indicating that gravity does not impact fluid behavior within tanks as hypothesized.

Upon confirming that gravity does not impact slosh testing, the simulations included in the metallic diaphragm testing and the flexible diaphragm testing are performed in a standard $1 \mathrm{G}$ environment. As with the freesurface simulations, the fluid behavior is analyzed in CFX-Post. The research investigation studies the CFD output, looking for any interesting or unique fluid behavior which could potentially have an effect on the propellant tank.

\footnotetext{
${ }^{\mathrm{x}}$ Future research creates spring, mass, damper analogs and pendulum analogs to simply model the fuel slosh scenarios tested in this research. The damping ratio, $\zeta$, is a key parameter in developing those analogs.
} 


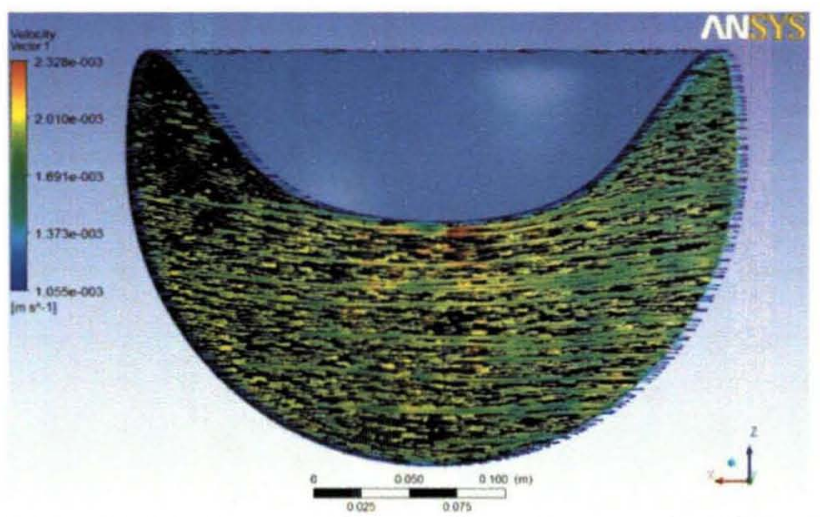

Figure 19. Velocity Vectors and Streamlines, Longitudinal Direction, $t=4 \mathrm{~s}$

The first parameter investigated during the rigid, crater-shaped diaphragm simulation is the velocity vectors during excitation. Referencing Figure 19, the velocity vectors during excitation remain in-plane in reference to the direction of excitation, the longitudinal direction (x-direction). Periodically throughout the simulation, however, some irregular behavior is noted to occur. Approximately every 50 timesteps, the velocity vectors abruptly change from uniform motion to erratic, seemingly random motion. Closer inspection indicates the presence of sub-surface vortices quickly forming and then disappearing. The fluid behavior during this vortex motion is analyzed to determine if the vortices pose a threat to the tank during lateral excitation.

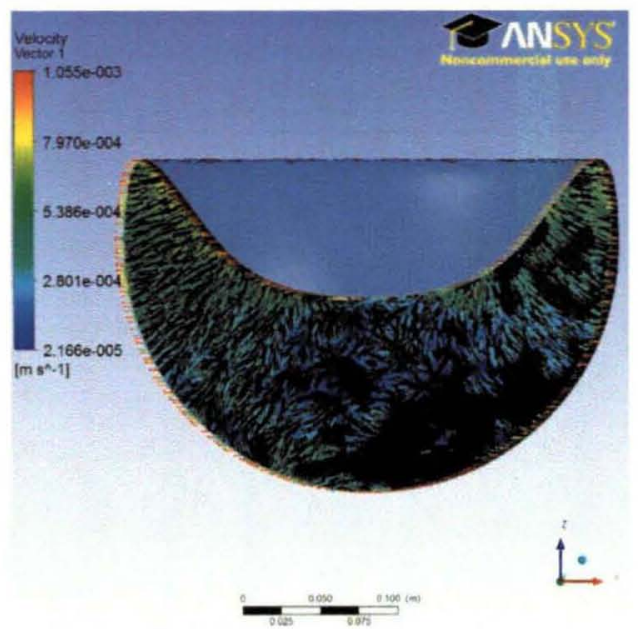

Figure 20. Velocity Vector Vortices, $t=16.05 s$

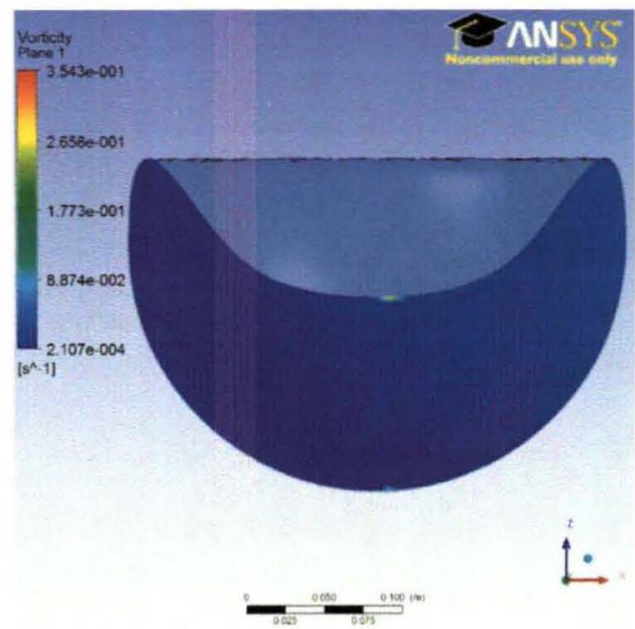

Figure 21. Vorticity Contours, $\mathrm{t}=\mathbf{1 6 . 0 5 \mathrm { s }}$

The sub-surface vortices are noted to occur periodically as the tank changes its direction of motion during excitation. As the tank changes excitation direction, the velocity vectors of the fluid consequently change direction in the time steps following the tank's direction change. However, some velocity vectors are still in the direction of the previous motion and thus oppose the velocity vectors in the new direction of motion. Velocity vectors oriented in two opposing directions interact with one another by creating this swirling motion as illustrated in Figure 20 . The velocity vectors in the middle of the tank seemingly originate from a random point and are directed in all directions $360^{\circ}$ around the point. To confirm the presence of this swirling motion, the vorticity of the fluid is examined.

Vorticity is defined as the curl of the fluid's velocity and is a vector quantity oriented along the fluid's axis of rotation. Mathematically, vorticity ${ }^{\mathrm{xi}}, y$, can be represented by the following equation where $v$ is the velocity of the fluid. $^{12}$

$$
y=\nabla \times v
$$

\footnotetext{
${ }^{\mathrm{xi}}$ Normally, vorticity is represented by $\omega$, but this research considers $\omega$ to represent natural frequency. To avoid conflict, vorticity is denoted by " $y$ ".
} 
Vorticity is an important parameter used to determine areas of a fluid field in which velocity vortices can form. Figure 21 illustrates the vorticity present in the fluid system at the same time the velocity vectors appear to have some type of non-uniform motion. It is noted that the vorticity in the fluid is 0 everywhere except for a small area at the bottom of the crater-shape and a smaller area at the bottom of the tank. The vorticity as seen in the XZ plane is inconclusive to determine if vortices are forming in the fluid domain.

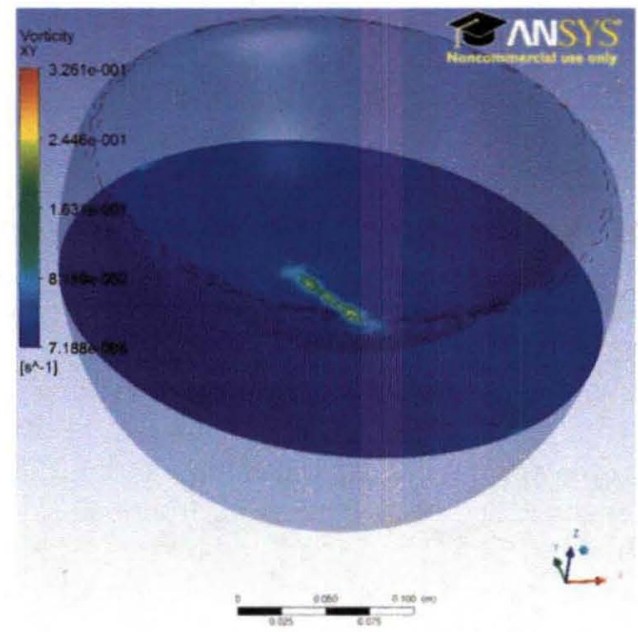

Figure 22. Isometric View of Vorticity, $t=16.05 \mathrm{~s}$

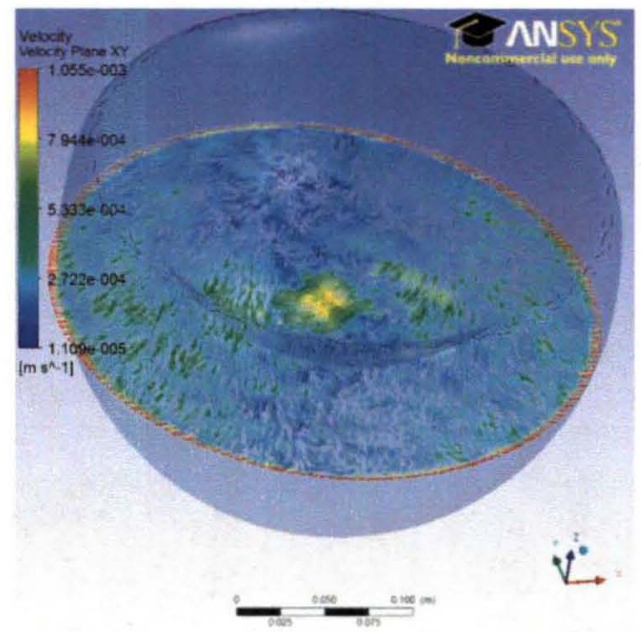

Figure 23. Isometric View of Velocity Vectors, $t=16.05 \mathrm{~s}$

Figure 22 is an alternative view of the vorticity in the fluid domain examined in the XY plane. This view clearly illustrates the area of high vorticity in the center of the tank right below the minimum point in the cratershape. This area of vorticity indicates a high fluid velocity which should furthermore produce noticeable vortices. Figure 23 illustrates the velocity vectors in the same XY plane at the same time step as Figure 22. It is noted that large velocity vectors, indicated in yellow, exist in the same area as the high vorticity. Additionally, vortices are present in the $+\mathrm{Y}$ and $-\mathrm{Y}$ directions away from the large velocity vectors as expected. Initially, it is concluded that these vortices are partially responsible for the tangential force responses in the longitudinal direction. Future testing will be able to validate this conclusion.

Understanding the fluid behavior during excitation is critical as any adverse forces or moments have the potential to be at their maximum values during this time period. However, understanding fluid behavior during the damping period is just as important to prevent any adverse internal effects on the tank.

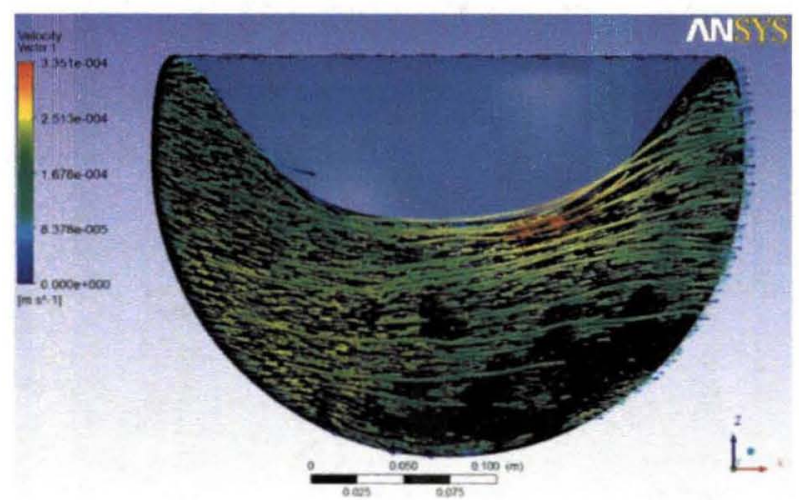

Figure 24. Velocity Vectors and Streamlines, Longitudinal Direction, $t=27 \mathrm{~s}$

Figure 24 illustrates the velocity vectors in the $\mathrm{X}-\mathrm{Z}$ plane during the seven second damping period from simulation time $20 \mathrm{~s}$ to $27 \mathrm{~s}$. It is noted that the velocity vectors during damping are not completely horizontal but instead follow more the contours on the tank itself. These velocity vector profiles can be explained by considering the coefficients of kinetic and static friction of a material, $\mu_{\mathrm{k}}$ and $\mu_{\mathrm{s}}$ respectively.

All simulations performed for the rigid crater-shape diaphragm incorporate the no-slip condition between the fluid and the sidewalls of the tank. Thus, all damping is a function of the viscous forces which arise as a result of 
the frictional forces along the sidewalls of the tank. In these simulations, friction forces are defined by the fluid's normal force and the coefficient of either static or kinetic friction depending on the state of the fluid. For most materials, the coefficient of static friction is significantly greater than the coefficient of kinetic friction. During the period of excitation, the frictional force experienced by the fluid is more a function of the coefficient of kinetic friction rather than the coefficient of static friction. On the contrary, during the period of damping, the fluid is moving at a slower velocity than during the excitation period. The frictional force experienced during damping is thus more a function of the coefficient of static friction than of the coefficient of kinetic friction.

Comparing the two periods, the frictional force experienced by the fluid during excitation is less than the frictional force experienced during damping. This allows the fluid to remain detached from the sidewalls of the tank during excitation and maintain horizontal velocity vectors. However, due to a larger frictional force during damping, the fluid does not have enough energy to overcome the frictional force experienced at the sidewalls and thus remains more attached to the sidewalls. This forces the velocity vectors to follow the contours of the tank during damping.

Upon completing analysis of the simulation involving the rigid, crater-shaped diaphragm tank, the only potential adverse behavior noted is the presence of the sub-surface vortices periodically occurring within the fluid. To overcome these sub-surface waves, a tank is designed in CATIA to have raised ridges, called asperities, lining the inside walls of the tank. The purpose of the asperities is to disrupt the swirling motion of the fluid within the tank and restore the velocity vectors to uniform motion. There are two different types of asperities tested to determine which, if either, are effective in eliminating sub-surface waves, while maintaining fluid damping during excitation. The first type of asperity is known as the linear asperity and spans the length of the inner wall from one side of the tank to the other side. The second type of asperity tested is hemispherical in nature and is incorporated along the inner tank walls in an organized manner. The same simulation parameters used in the rigid, crater-shaped diaphragm simulation are also used in these simulations to determine the effectiveness of the asperities.

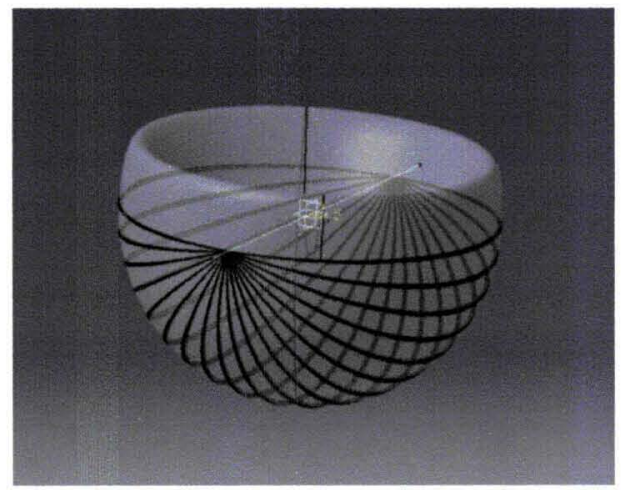

Figure 25. Rigid, Crater-Shaped Diaphragm with Linear Asperities

Figure 25 illustrates the rigid, crater-shaped propellant tank implemented with the linear asperities. There are two types of testing performed with this tank: in-plane testing, meaning the direction of excitation is in the same plane as the orientation of the asperities, or out-of-plane testing, meaning the direction of excitation is perpendicular to the orientation of the asperities. The in-plane case is tested first. 


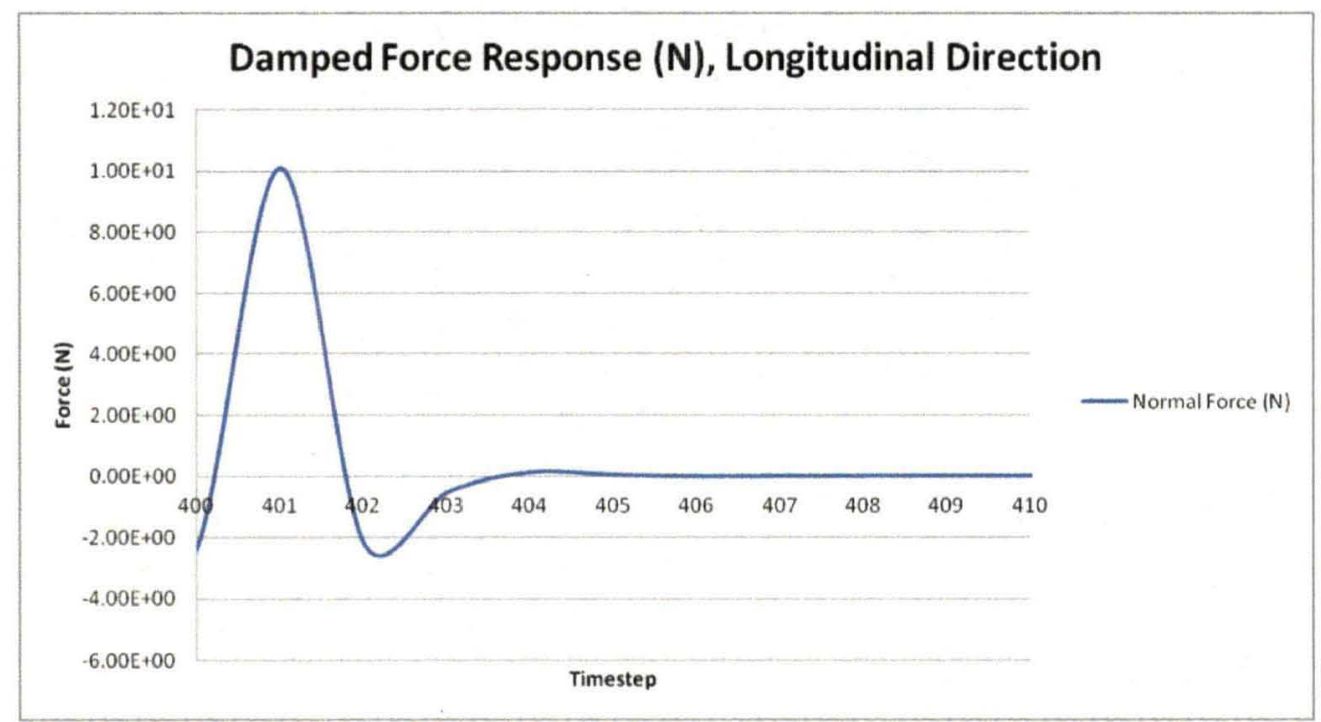

Figure 26. Rigid Crater-Shaped Diaphragm, Linear Asperities, In-Plane Damped Force Response, Longitudinal Direction

Figure 26 is the damped force response of the tank with linear asperities excited in-plane. Similar to the rigid, crater-shaped diaphragm simulation, the fluid within the tank dampens completely within 4 timesteps. Again, the highly damped simulation becomes difficult to analyze because of the lack of data and lack of peak-to-peak points. The data during damping is input into the user-defined MATLAB algorithm to solve the complex eigenvalue problem. Similar to the rigid, crater-shaped diaphragm tank analysis, the data from the reaction moments are used as the second degree of freedom in the eigenvalue problem. The results of the eigenvalue algorithm are displayed in Table 3 .

Table 3. Rigid, Crater-Shaped Diaphragm with Linear Asperities Damping Results, In-Plane

\begin{tabular}{|c|c|}
\hline Parameter & Value \\
\hline$\omega_{\mathrm{n}}(\mathrm{rad} / \mathrm{s})$ & 8.6330 \\
\hline$\omega_{\mathrm{d}}(\mathrm{rad} / \mathrm{s})$ & 8.1008 \\
\hline$\zeta$ & 0.3457 \\
\hline
\end{tabular}

Table 3 presents the damping results taken from the 2-DOF eigenvalue problem computed by MATLAB. The undamped natural frequency of the tank with asperities is $1.3739 \mathrm{~Hz}$, upon conversion, and the damped natural frequency of the system is $1.2893 \mathrm{~Hz}$. These natural frequency values are very close to the natural frequency values of the rigid, crater-shaped diaphragm tank without asperities. It can therefore be concluded that the addition of asperities within the tank does not change the natural frequency of the system by a significant amount.

MATLAB also generates the damping ratio, $\zeta$, of this system from the coupled, damped force response data. The value of 0.3457 is a useful parameter for alternative modeling of this system but like the rigid, cratershaped diaphragm system, it is not applicable in this part of the research at this point. This research is concerned with obtaining the damping ratio of the force response curve, $\zeta_{\mathrm{F}}$. However, due to the lack of data and peaks in the force response curve of Figure 32, it is not possible to obtain a curve-fit to this data to extract a damping ratio value. Therefore, the damping ratio of the force response curve cannot be found at this time. 


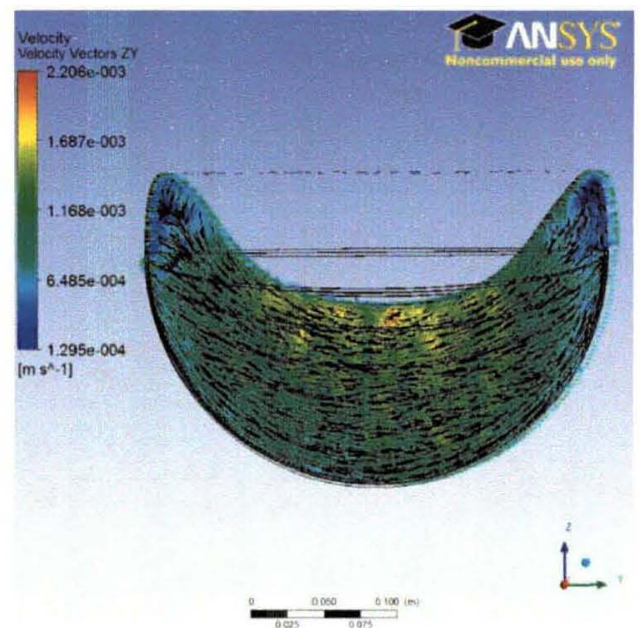

Figure 27. Velocity Vectors, ZY Plane, $t=8.55 \mathrm{~s}$

The analysis begins by referencing the points in time during the original simulation (involving the tank without asperities) when the vortices occur. Figure 27 illustrates the velocity vectors at the $171^{\text {st }}$ timestep $(\mathrm{t}=8.55 \mathrm{~s})$ which corresponds to a timestep in the rigid, crater-diaphragm tank simulation where vortices were noted as occurring. It is noted that no such vortices occur at this timestep, or any other timestep corresponding to the presence of vortices from the original simulation. A further examination into other timesteps in this analysis shows no indication that sub-surface vortices exist. It can therefore be initially concluded that the asperities function as intended.

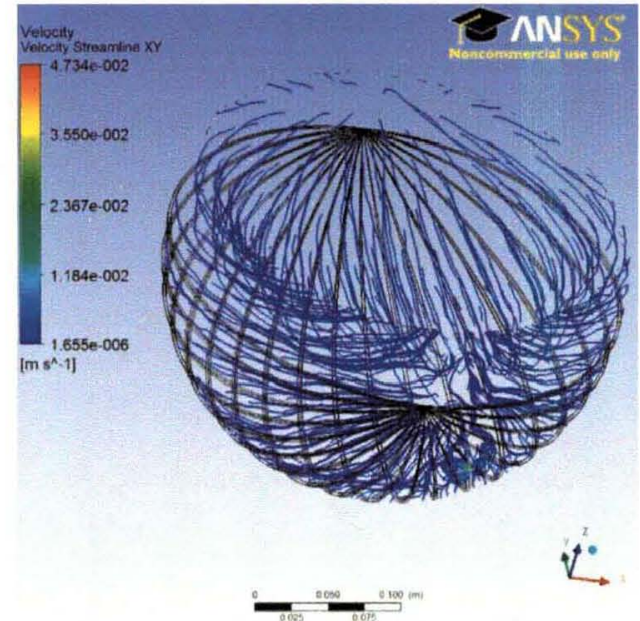

Figure 28. Velocity Streamlines, Iso. View, $t=8.55$

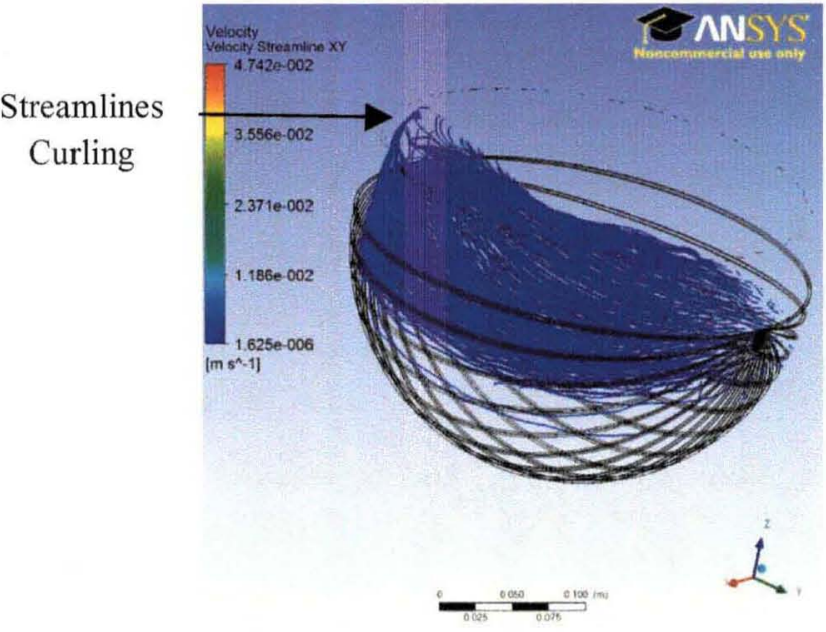

Figure 29. Velocity Streamlines, Iso. View, $t=8.55 \mathrm{~s}$

A further analysis into Figure 27 raises questions as to the behavior of the fluid in the volume of the tank above the asperities. Examining the velocity vectors in this volume indicates a decrease in velocity magnitude and apparent disorder among the vector orientations. Figure 28 presents an alternative view of the velocity vectors by illustrating the behavior of the velocity streamlines within the entire tank. Examining this alternative view at the $171^{\text {st }}$ timestep indicates that there is in fact order among the streamlines in the tank; possibly as a result of the asperities. The streamlines converge on the centerline midpoint which is the same location in the tank where the asperities join together and from which they originate. Figure 29 further illustrates the organization of the streamlines.

Figure 29 is created by considering a plane perpendicular to the ZY plane illustrated in Figure 27. By creating an $\mathrm{XY}$ plane and locating it tangent to the low-point of the crater-shaped diaphragm, the streamlines within this plane help to better explain what is happening in Figure 27. The portion of the tank above the asperities is subjected to nearly vertical velocity streamlines at the midpoint of the tank's centerline. Figure 29 seems to suggest that this simulation is a "free-surface" model with the fluid exhibiting this "sloshing-like" behavior. However, 
because it is known that the inside of the tank is void of air, an alternative explanation must accompany this Figure. Examining Figure 29 closely, it is noted that the velocity streamlines begin to curl around the curved diaphragm surface in contact with the fluid. If these streamlines are extended, it would be evident there exists swirling motion in this portion of the tank where asperities do not exist. Unfortunately, placing asperities in this portion of the model would be inaccurate as this part of the tank is the metallic diaphragm in laboratory testing. Incorporating asperities along the inner walls of the metallic diaphragm is a possibility but it is not known if current metallic diaphragms are designed with such structures. Until further investigation is performed into metallic diaphragms, the current computational model remains unchanged.

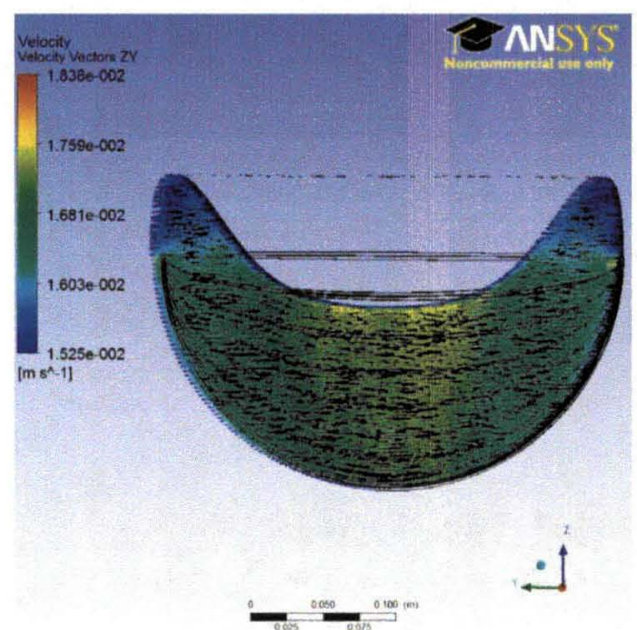

Figure 30. Velocity Vectors, $Z Y$ Plane, $t=4.65 s$

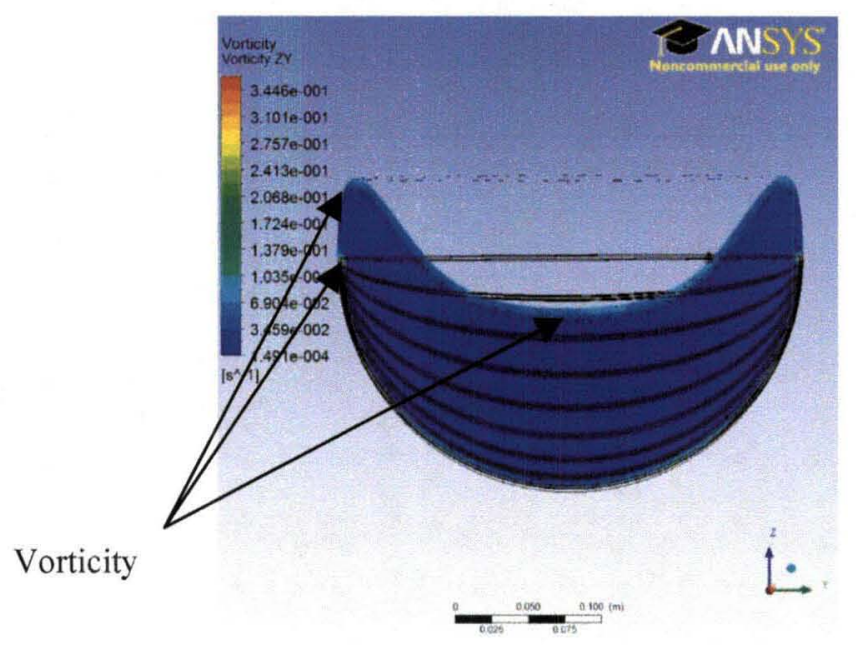

Figure 31. Vorticity Contours, ZY Plane, $t=4.65 \mathrm{~s}$

Initial conclusions about this simulation suggest that the asperities function as intended and eliminate subsurface vortices. Figure 30 illustrates the velocity vectors at the $93^{\text {rd }}$ timestep corresponding to $4.65 \mathrm{~s}$ into the simulation. Examining these vectors, there is nothing unusual about their behavior or orientation to suggest the presence of sub-surface vortices. To validate this assertion, the vorticity of the fluid is analyzed to clarify no vortices exist. Figure 31 illustrates the vorticity in the ZY plane which is the plane of excitation of the tank. This illustration, captured at the $93^{\text {rd }}$ timestep, indicates small areas of vorticity below the crater-shaped diaphragm, at the midpoint of the centerline of the tank as well as small amounts of yorticity in the upper volume of the tank above the asperities. These three regions are further examined to determine to what extent the magnitude of vorticity affects the tank system.

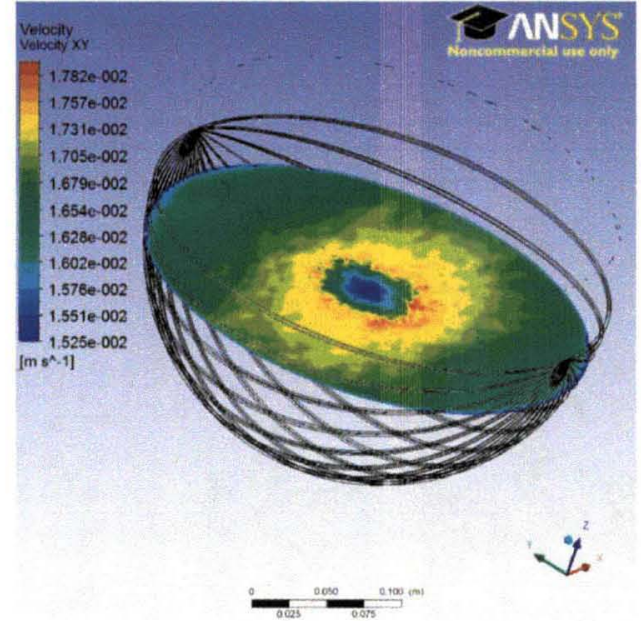

Figure 32. Velocity Contours, XY Plane, $t=4.65 \mathrm{~s}$

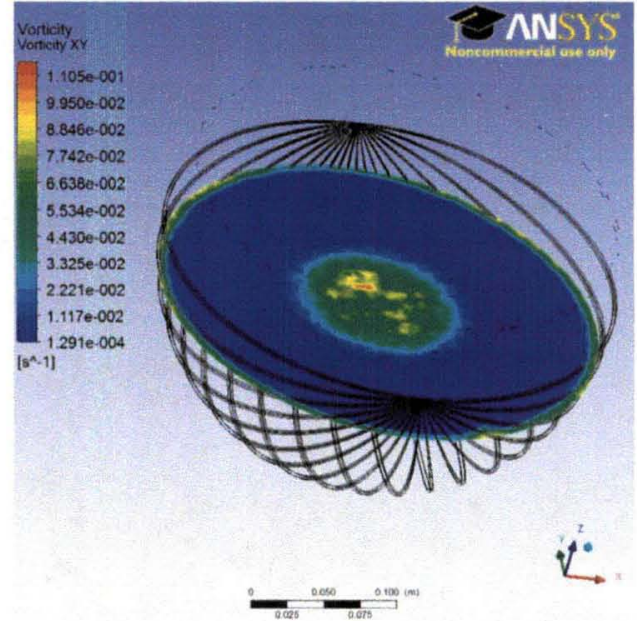

Figure 33. Vorticity Contours, XY Plane, $t=4.65 \mathrm{~s}$

When considering the vorticity of the fluid in a particular region of the tank, it is helpful to include the velocity contours or streamlines as a basis for backing conclusions drawn when analyzing vorticity contours. 
Examining Figure 32, it is noted that there is somewhat of a uniform velocity throughout the tank except in the middle of the plane which corresponds to the area of the tank below the low-point of the crater-shaped diaphragm. This circular region of varying velocities could potentially be an area where vortices form. Referring to Figure 33, the presence of vorticity is noted in the same area as the varying velocities. However, the magnitude of this vorticity is not a cause for concern as to the propagation of potentially dangerous sub-surface vortices.

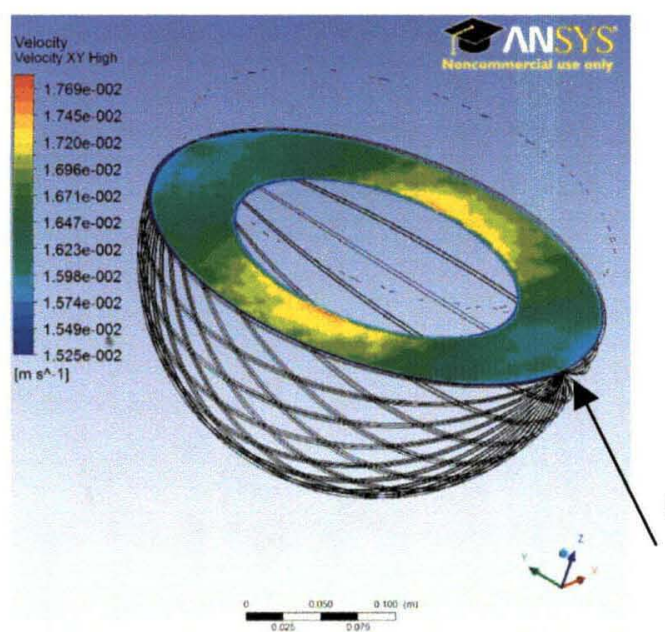

Figure 34. Vel. Contours, XY Plane, $t=4.65 \mathrm{~s}$

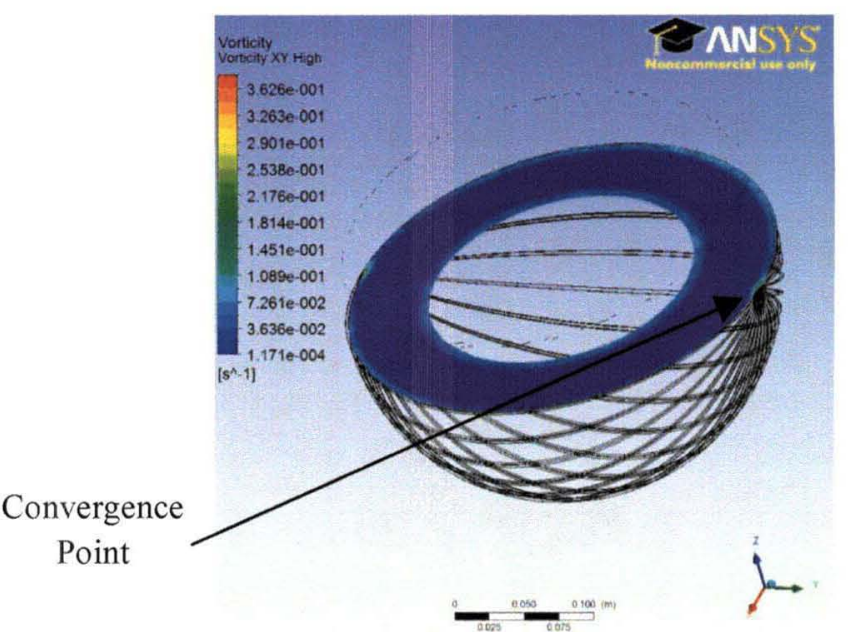

Figure 35. Vorticity Contours, XY Plane, $t=4.65 \mathrm{~s}$

Figures 34 and 35 are taken from the XY plane created at the centerline of the tank. From Figure 31 , vorticity is indicated at the midpoint of the tank's centerline where the asperities converge. Considering Figure 34 first, the velocity contours in this plane are nearly symmetric, with the higher velocities being located in the middle of the plane around the crater-shaped diaphragm. Examining the convergence point of the asperities, it is noted that the velocity contours are relatively lower in magnitude than other areas within the plane and there is approximately a single velocity magnitude in the immediate area surrounding the convergence point. With this in mind, it should be expected that the vorticity experienced at this point is small and insignificant. Referring to Figure 35 , the vorticity at the convergence point of the asperities is minimal in size. However, when examining the magnitude of the vorticity at this point, it is noted that the vorticity is one order of magnitude higher than the vorticities examined in Figure 33. This can be attributed to the intersection of multiple velocity streamlines from multiple directions as illustrated in Figure 28. At this point, the magnitude of vorticity is not deemed significant enough to impact the tank system. However, future studies regarding the impacts of asperities within propellant tanks may need to reconsider the convergence point of the asperities.

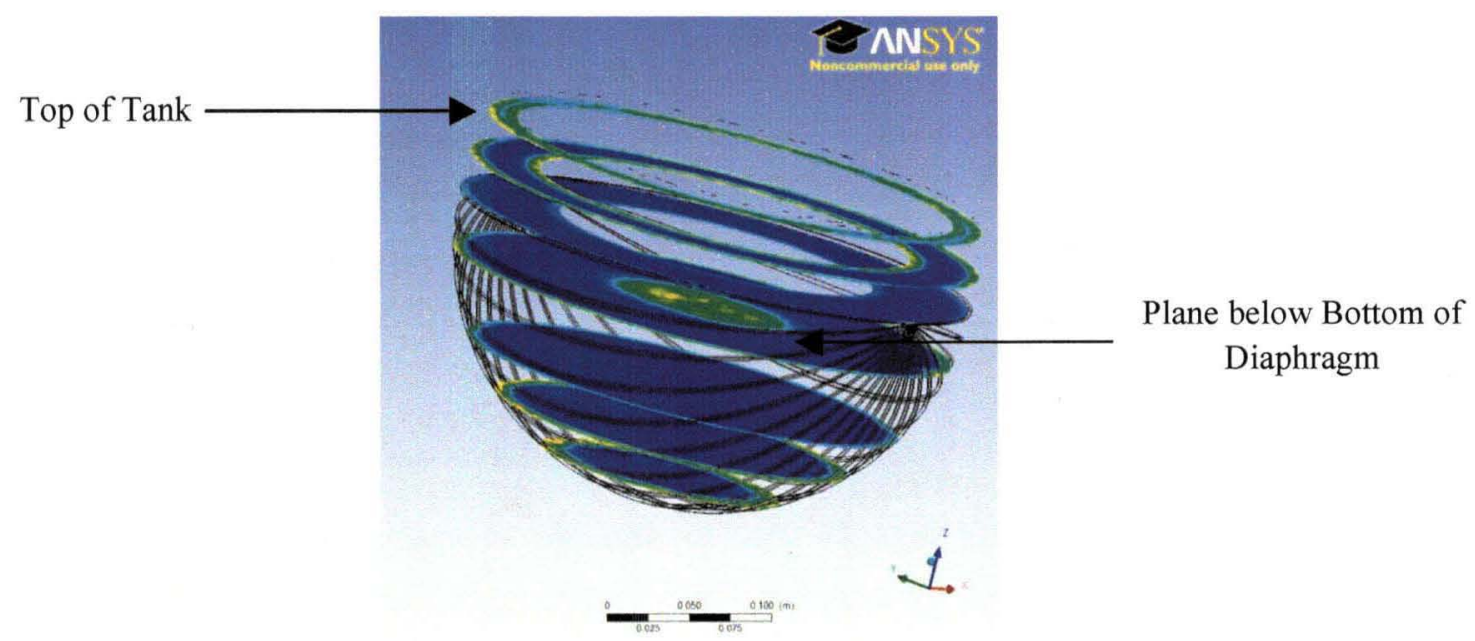

Figure 36. Vorticity Contours, Various XY Planes, $t=4.65 \mathrm{~s}$ 
The third region of the tank under vorticity analysis is the top volume of the tank above the asperities. It has already been observed from Figure 29 that there are curling velocity streamlines in the upper portion of the tank. Curling velocity streamlines are a good indication as to the potential of vorticity being present. Referring to Figure 36 , the uppermost plane is located at the top-most portion of the tank where the curling velocity streamlines are located. As indicated by the contours in this plane, vorticity clearly exists in this region of the tank. Due to the multiple vorticity planes illustrated in this post-process view, an accurate legend cannot be created to satisfy all of the contours. The vorticity in the upper region is of the same magnitude as the vorticity just below the crater-shaped diaphragm. Unlike the vorticity below the diaphragm, the vorticity in the upper plane could have the potential to negatively impact the system. With the curling velocity streamlines being confined to a small volume in the upper portion of the tank, their interactions with the diaphragm walls are bound to be more prevalent and thus, a greater reaction force on the diaphragm may exist. With potentially larger forces in this region, far from the center of mass of the system, there exists the potential of large, adverse moments originating in this region of the tank. Therefore, with further study of metallic diaphragms, these potentially large forces and moments can be minimized or eliminated so as to not pose a negative impact on the tank.

The addition of the linear asperities results in the tank becoming asymmetric. Thus, additional simulations are required to consider alternative orientations of the tank during excitation. The next simulation performed considers the same tank system outfitted with linear asperities. However, the direction of excitation of this new setup is in a plane perpendicular to the orientation of the asperities. Again, the damping parameters are calculated to determine the impact of the tank orientation on damping and natural frequency values. Furthermore, the fluid behavior is analyzed, taking into consideration velocity vectors, velocity streamlines and vorticity.

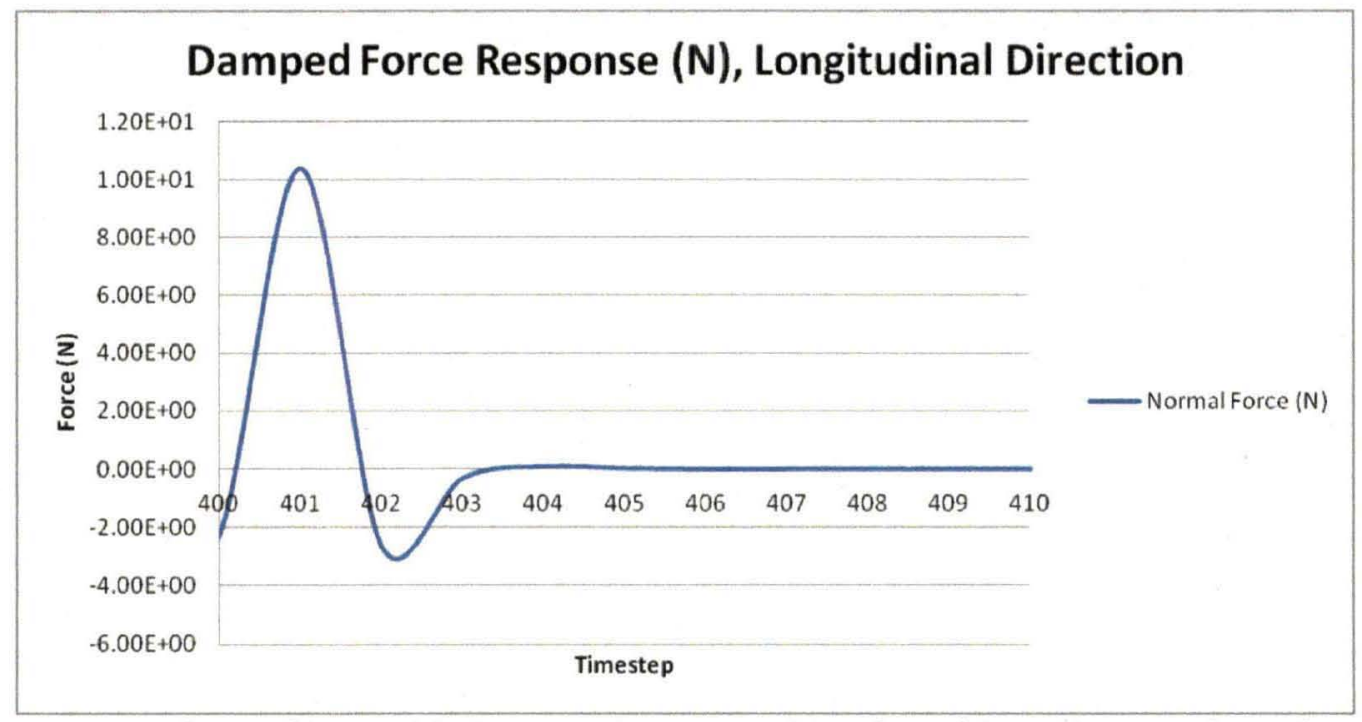

Figure 37. Rigid Crater-Shaped Diaphragm, Linear Asperities, Out-of-Plane Damped Force Response, Longitudinal Direction

Figure 37 is the damped force response of the tank with linear asperities excited out-of-plane. Similar to past simulations, the fluid within the tank dampens completely within 4 timesteps. The highly damped simulation becomes difficult to analyze because of the lack of data and lack of peak-to-peak points. Following past simulations, the damping data is input into the user-defined MATLAB algorithm to solve the complex eigenvalue problem. The data from the reaction moments are used as the second degree of freedom in this eigenvalue problem. The results of the eigenvalue algorithm are displayed in Table 4.

Table 4. Rigid, Crater-Shaped Diaphragm with Linear Asperities Damping Results, Out-of-Plane

\begin{tabular}{|c|c|}
\hline Parameter & Value \\
\hline$\omega_{\mathrm{n}}(\mathrm{rad} / \mathrm{s})$ & 8.6342 \\
\hline$\omega_{\mathrm{d}}(\mathrm{rad} / \mathrm{s})$ & 8.0412 \\
\hline$\zeta$ & 0.3642 \\
\hline
\end{tabular}


Table 4 presents the damping results taken from the 2-DOF eigenvalue problem computed by MATLAB. The undamped natural frequency of the tank with asperities out-of-plane is $1.3742 \mathrm{~Hz}$, upon conversion, and the damped natural frequency of the system is $1.2798 \mathrm{~Hz}$. These natural frequency values are very close to the natural frequency values of both the rigid, crater-shaped diaphragm tank without asperities and the tank with asperities excited in-plane.

MATLAB also generates the damping ratio, $\zeta$, of this system from the coupled, damped force response data. The value of 0.3642 is a useful parameter for alternative modeling of this system but like the rigid, cratershaped diaphragm system, it is not applicable in this part of the research at this point. However, due to the lack of data and peaks in the force response curve of Figure 45, it is not possible to obtain a curve-fit to this data to extract a damping ratio value, $\zeta_{\mathrm{F}}$. Therefore, the damping ratio of the force response curve cannot be found at this time.

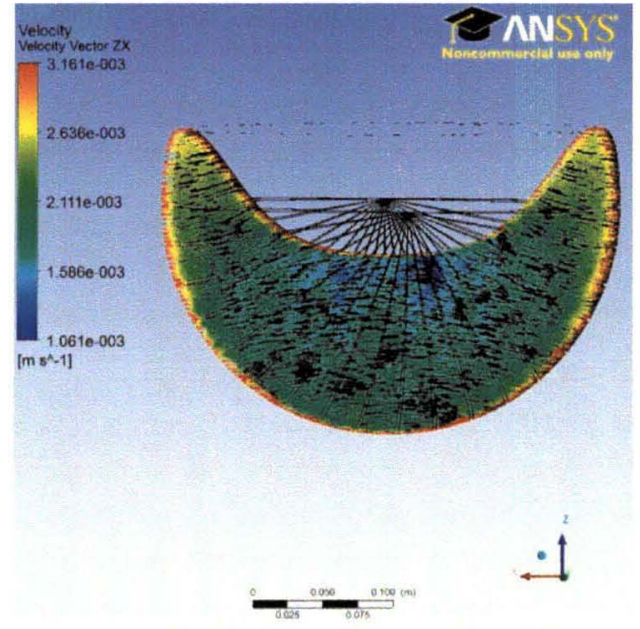

Figure 38. Velocity Vectors, ZX Plane, $t=8.10 \mathrm{~s}$

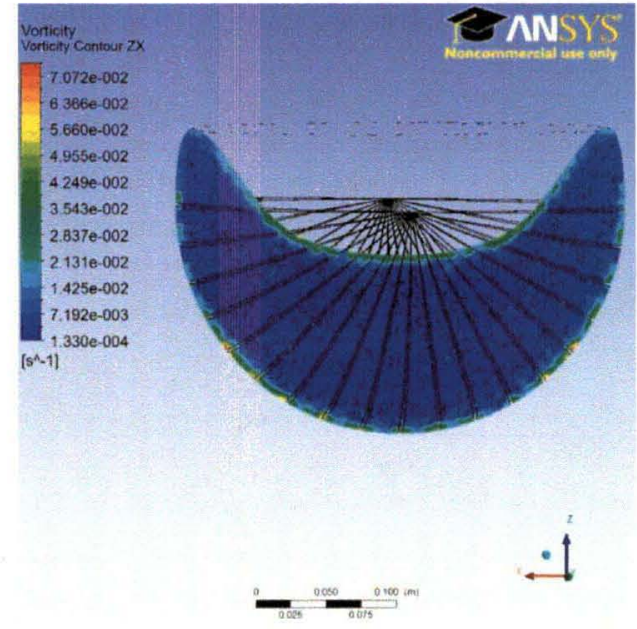

Figure 39. Vorticity Contours, $\mathrm{ZX}$ Plane, $\mathrm{t}=\mathbf{8 . 1 0 \mathrm { s }}$

The asymmetry of the tank as a result of the addition of the asperities causes an investigation into the fluid behavior during possible orientations of the tank in a real spacecraft fuel slosh scenario. As with the in-plane scenario, the timesteps from the original asperity-free tank simulation which caused sub-surface vortices are investigated in this simulation to determine if the out-of-plane asperities are effective in suppressing these adverse conditions.

Following past simulation analysis, sub-surface vortices are observed as occurring at specific, periodic timesteps throughout the simulation. For this simulation, the $162^{\text {nd }}$ timestep is the timestep where sub-surface vortices should manifest themselves. Figure 38 is an output at the $162^{\text {nd }}$ timestep, 8.10 s into the excitation, of the velocity vectors in the $\mathrm{ZX}$ plane which is the plane of excitation. It is noted that the velocity vectors are parallel to the direction of excitation and there is no indication of vortices forming. The magnitude of the fluid velocity is at its highest in this timestep along the walls of the tank where out-of-plane asperities exist. Yet, regardless of increased velocity magnitude, no vortices appear to be forming at these locations. This assertion is validated by considering Figure 47 , the vorticity contours in the ZX plane at the $162^{\text {nd }}$ timestep.

Figure 39 indicates the presence of vorticity in the tank at this timestep. Compared to the vorticity when vortices exist, the magnitude of the vorticity in Figure 47 is one order of magnitude less. The greatest vorticity at the $162^{\text {nd }}$ timestep is located along the asperities. It is expected that vorticity exists here as the extruding tank structures disrupt the otherwise uniform fluid flow. The vorticity, however, is not great enough to generate sub-surface vortices since they do not appear in Figure 38 at the locations of the asperities. Additionally, there is no indication that vortices appear in any other time step in this simulation. Thus, it can be initially concluded that the orientation of the asperities has no measureable impact on the generation of sub-surface vortices. Furthermore, the out-of-plane asperities function as intended and eliminate the presence of sub-surface vortices. 


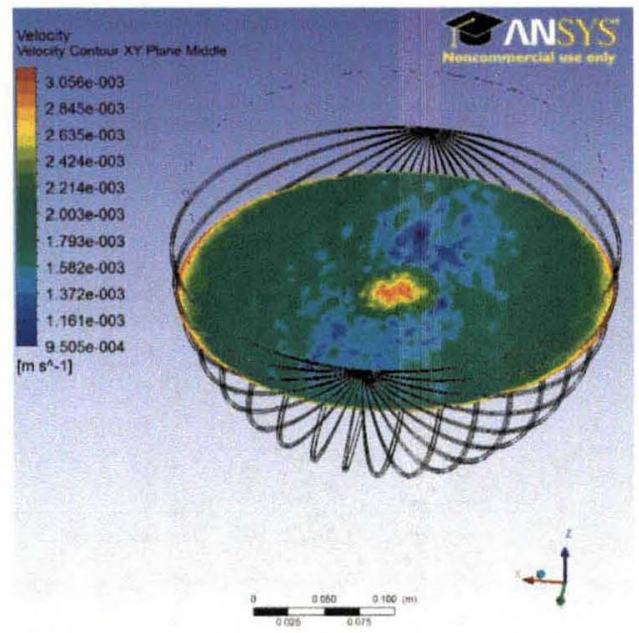

Figure 40. Velocity Contours, XY Plane, $t=8.10$

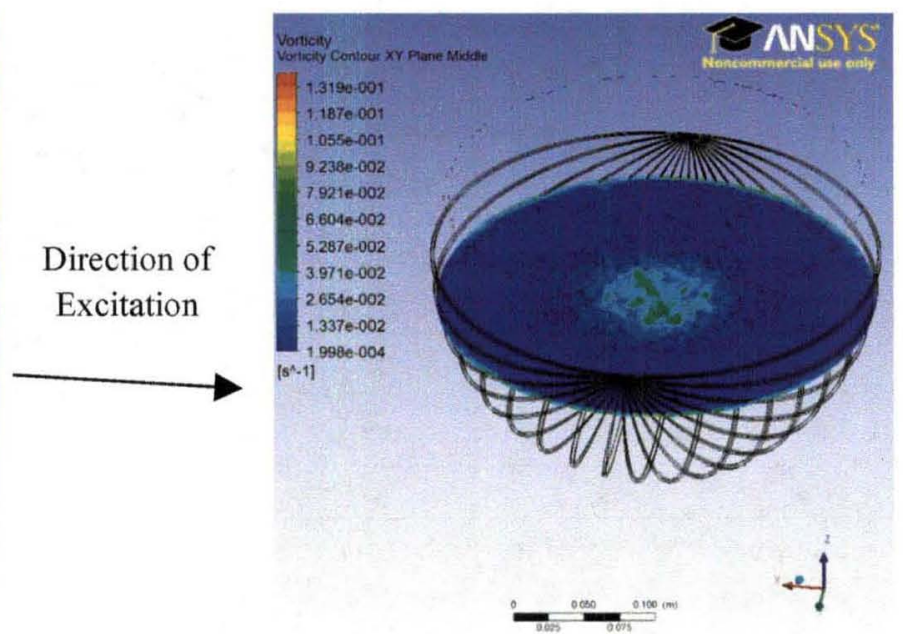

Figure 41. Vorticity Contours, XY Plane, $t=8.10 \mathrm{~s}$

It is important to further consider Figure 39 and investigate other locations highlighted in the ZX plane where vorticity exists. Noting that there is a small occurrence of vorticity in the upper middle of the ZX plane just under the diaphragm, vorticity in alternative planes is analyzed to better understand where this vorticity is and how it is behaving. Figure 40 is a look into the velocity contours in an XY plane located tangent to the lower surface of the crater-shaped diaphragm. Again, the velocity magnitudes are higher at the edge of the plane than any other location in the plane. This suggests the asperities are accelerating the fluid flow across the entire plane but not to the extent of forming vortices. The other location of high velocity is occurring at the center of the plane which corresponds to the area just below the surface of the low point in the crater diaphragm. As the fluid interacts with areas of the diaphragm above this, the fluid is forced down and around the diaphragm and into a smaller cross-sectional area (perpendicular to the direction of fluid flow) of the tank before passing the mid-point of the tank and back into a larger cross-sectional area. This smaller cross-sectional area is believed to be directly responsible for the increase in velocity.

Figure 41 considers vorticity contours in the same XY plane tangent to the low-point of the crater-shaped diaphragm. As with Figure 40, Figure 41 indicates vorticity along the outer edges of the tank along the asperities. This vorticity is small in magnitude and is not significant enough to generate sub-surface vortices. Additionally, a large amount of vorticity is located at the center of the plane just below the crater-shaped diaphragm. This vorticity is due to the interaction of the velocity vectors in this plane and the velocity vectors from upper parallel planes being forced in the $-\mathrm{Z}$ direction by the diaphragm. Although the cross-product of these two sets of vectors raises concern as to the occurrence of vortices, the magnitude of this vorticity is again small enough to dismiss the notion that vortices occur.

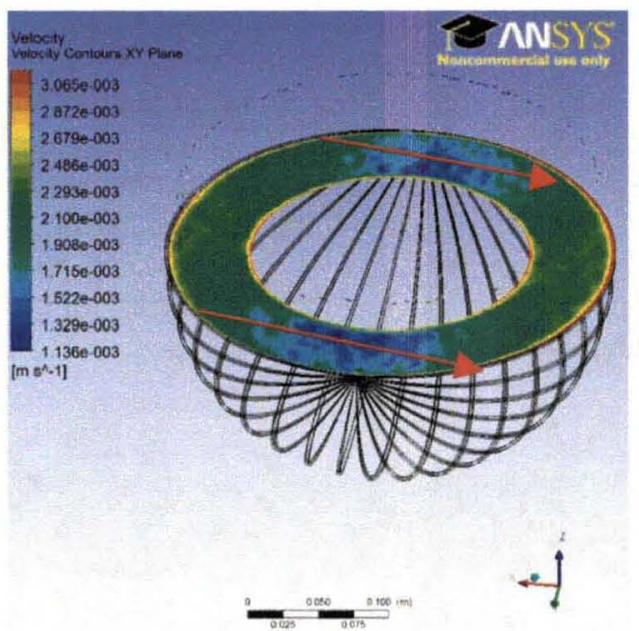

Figure 42. Velocity Contours, XY Plane, $t=8.10 \mathrm{~s}$

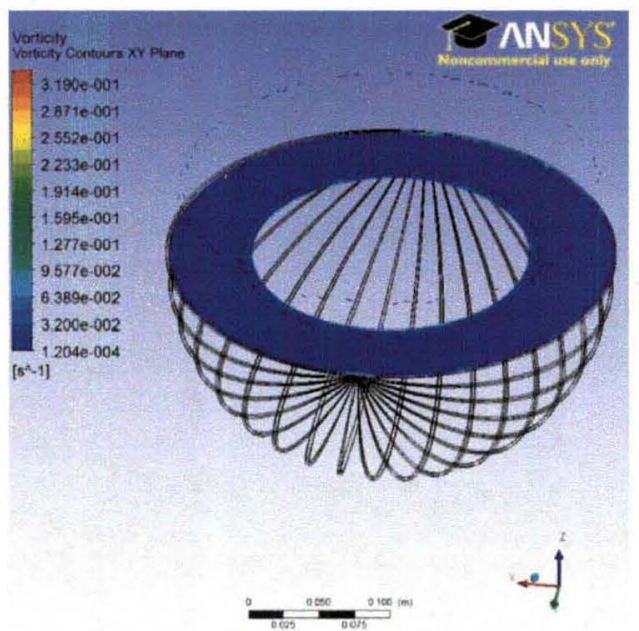

Figure 43. Vorticity Contours, XY Plane, $t=8.10 \mathrm{~s}$ 
The in-plane analysis investigation considered the velocities and vorticity in the plane corresponding to the location where the tank and diaphragm come together and asperities are no longer present. As such, the same plane is considered in this analysis. Figure 42 illustrates the velocity contours in the XY plane located at the top of the tank and below the diaphragm. The largest velocity magnitudes are located at the edge of the plane at the ends where the tank is shaking and also located in the middle of the tank surrounding the crater-shaped diaphragm. The velocity at the outer edges of the tank is still in contact with the last asperity and therefore, is expected to follow the observed trend of being the largest velocity in the plane. Additionally, the velocity contours surrounding the edge of the crater-shape are again expected to be the largest in this plane. These contours represent the velocity vectors being forced in the $-\mathrm{Z}$ direction because of the presence of the diaphragm. It is, however, interesting to note the lesser velocity contours surrounding the crater-shape that are uninhibited by the crater-shape. The velocity vectors are in the X-direction and as such, when vectors are drawn from one side of the tank to the other side, they do not interfere with the crater-shape on the outer edges of the crater as illustrated by the red arrows in Figure 42. It would be expected that these vectors either follow uniform velocity magnitudes with other areas of the tank (i.e. the green contours) or would have slightly higher magnitudes than other contours in the tank due to their continuous line from one side of the tank to the other. This interesting observation requires further investigation in subsequent simulations.

Figure 43 illustrates the vorticity contours in the same plane highlighted in Figure 42 . The vorticity in this plane is essentially non-existent except for small areas at the midpoint of the centerline of the tank where the asperities join together. The magnitudes of these vorticity contours at these locations are not a cause for concern. Additionally, should vortices have formed, the velocity contours in Figure 42 would have indicated such. Thus, it can be initially concluded that the presence of asperities, regardless of orientation, are effective in eliminating subsurface vortices.

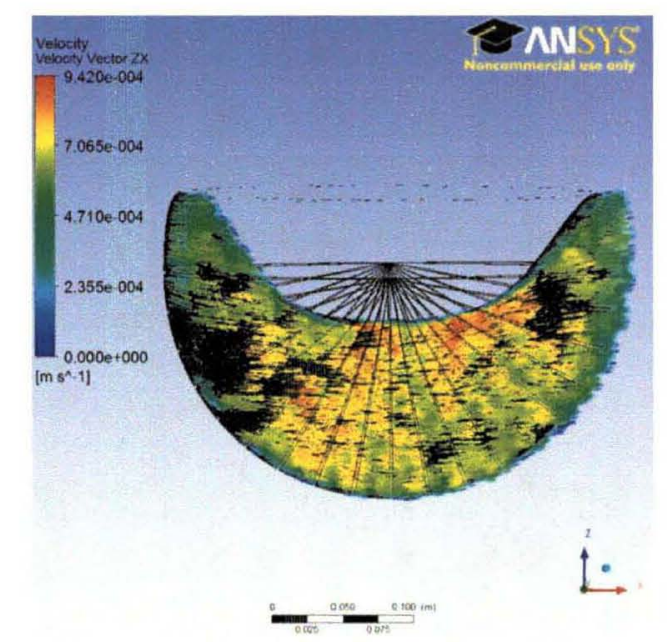

Figure 44. Velocity Vectors, ZX Plane, $t=21.0 s$

In order to complete the investigation into the effects of asperities on fluid behavior, the fluid damping for the out-of-plane case is investigated. Figure 44 illustrates the velocity vector contours 1 second into damping. It is important to note the magnitudes of the vectors. One second after excitation has ceased, the magnitudes of the velocity vectors have already decreased two orders of magnitude as compared to the magnitudes highlighted in Figure 38. Additionally, there are not many vectors with zero magnitude. The largest velocity vectors still exist directly below the low point of the crater-shaped diaphragm. The smallest velocity vectors are located in between the asperities. It is these vectors which are expected to go to zero magnitude first as damping continues. 


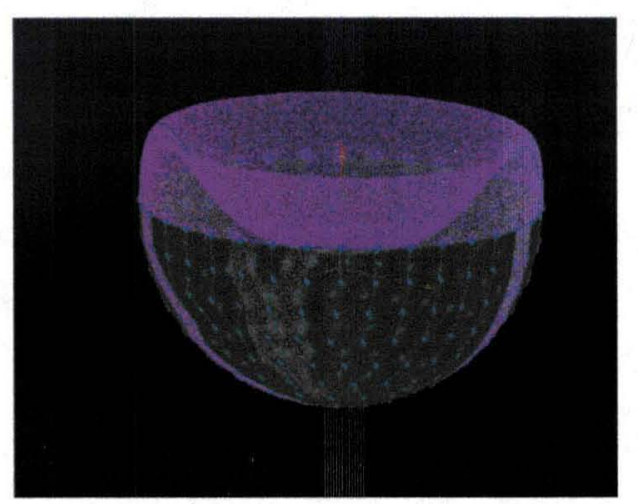

Figure 45. Meshed Computational Model of Rigid, Crater-Shaped Diaphragm with Spherical Asperities

The investigation into the impact of asperities on fluid behavior continues with an analysis of spherical asperities along the inner tank walls. Illustrated in Figure 45, the propellant tank is implemented with small, hemispherical ridges on the inside of the tank. These hemispheres extrude approximately $2 \mathrm{~mm}$ from the surface of the tank. This height is chosen so as to cause no interference between the asperities and the flexible diaphragm when the diaphragm is depleted ${ }^{\mathrm{xii}}$. The purpose of the spherical asperities is to build the test matrix of simulating alternative asperity shapes to determine an optimal shape (should one exist) for eliminating sub-surface vortices.

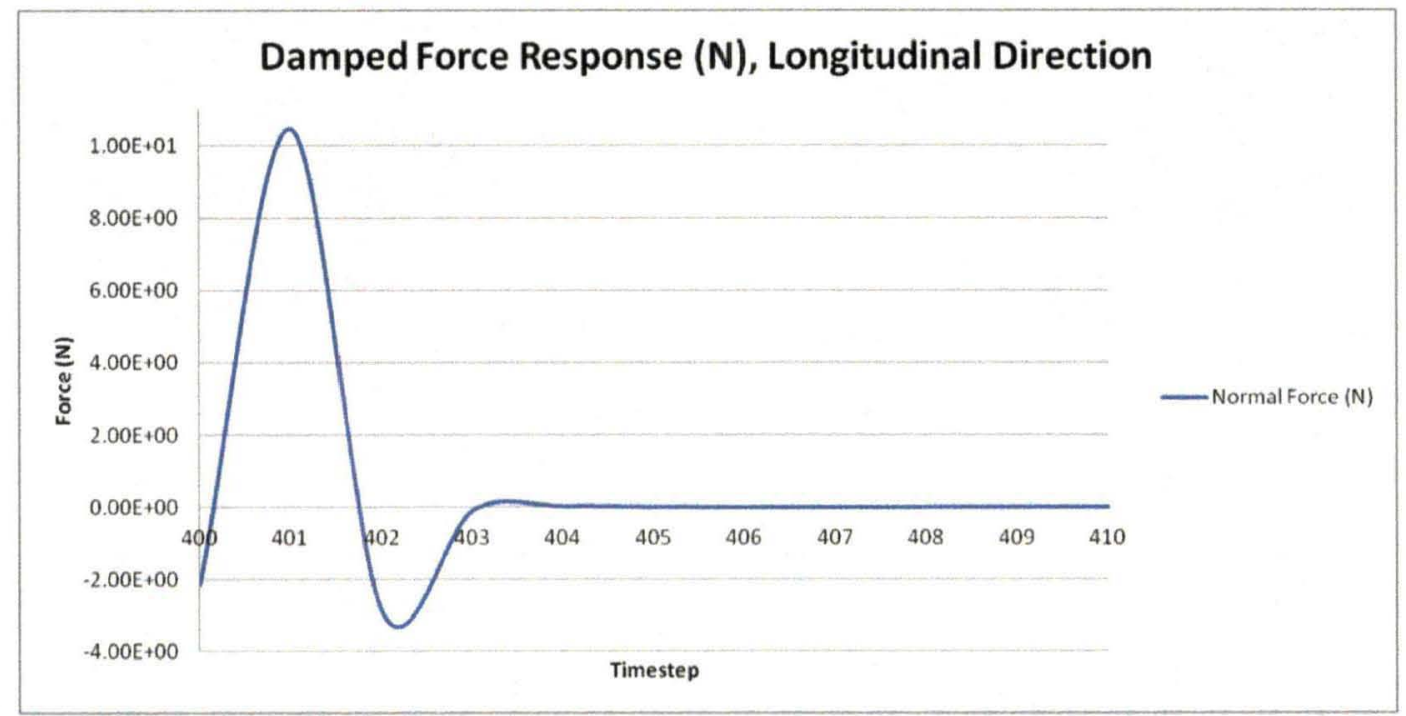

Figure 46. Rigid Crater-Shaped Diaphragm, Linear Asperities, Out-of-Plane Damped Force Response, Longitudinal Direction

Figure 46 is the damped force response of the tank implemented with spherical asperities. Similar to past simulations, the fluid within the tank dampens completely within 4 timesteps. The highly damped simulation becomes difficult to analyze because of the lack of data and lack of peak-to-peak points. Following past simulations, the damping data is input into the user-defined MATLAB algorithm to solve the complex eigenvalue problem. The data from the reaction moments are used as the second degree of freedom in this eigenvalue problem. The results of the eigenvalue algorithm are displayed in Table 5.

\footnotetext{
xii The current computational tank is not implemented with a flexible diaphragm. The dimensions of the asperities are based on the hypothetical scenario where a flexible diaphragm is implemented into the tank.
} 
Table 5. Rigid, Crater-Shaped Diaphragm with Spherical Asperities Damping Results

\begin{tabular}{|c|c|}
\hline Parameter & Value \\
\hline$\omega_{\mathrm{n}}(\mathrm{rad} / \mathrm{s})$ & 8.6421 \\
\hline$\omega_{\mathrm{d}}(\mathrm{rad} / \mathrm{s})$ & 7.8597 \\
\hline$\zeta$ & 0.4158 \\
\hline
\end{tabular}

Table 5 presents the damping results taken from the 2-DOF eigenvalue problem computed by MATLAB. The undamped natural frequency of the tank with spherical asperities is $1.3754 \mathrm{~Hz}$, upon conversion, and the damped natural frequency of the system is $1.2509 \mathrm{~Hz}$. These natural frequency values are very close to the natural frequency values of both the rigid, crater-shaped diaphragm tank without asperities and the tanks with asperities.

MATLAB also generates the damping ratio, $\zeta$, of this system from the coupled, damped force response data. The value of 0.4158 is a useful parameter for alternative modeling of this system but like the rigid, cratershaped diaphragm system, it is not applicable in this part of the research at this point. However, due to the lack of data and peaks in the force response curve of Figure 46, it is not possible to obtain a curve-fit to this data to extract a damping ratio value, $\zeta_{\mathrm{F}}$. Therefore, the damping ratio of the force response curve cannot be found at this time.

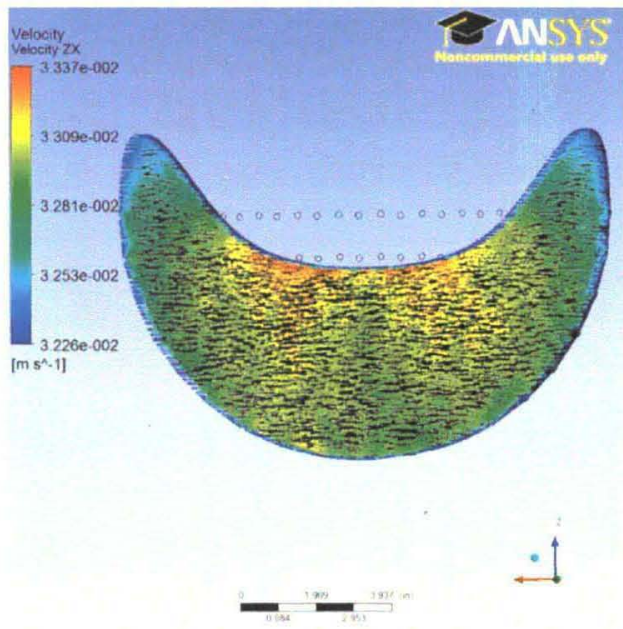

Figure 47. Velocity Vectors, ZX Plane, $t=5.50 \mathrm{~s}$

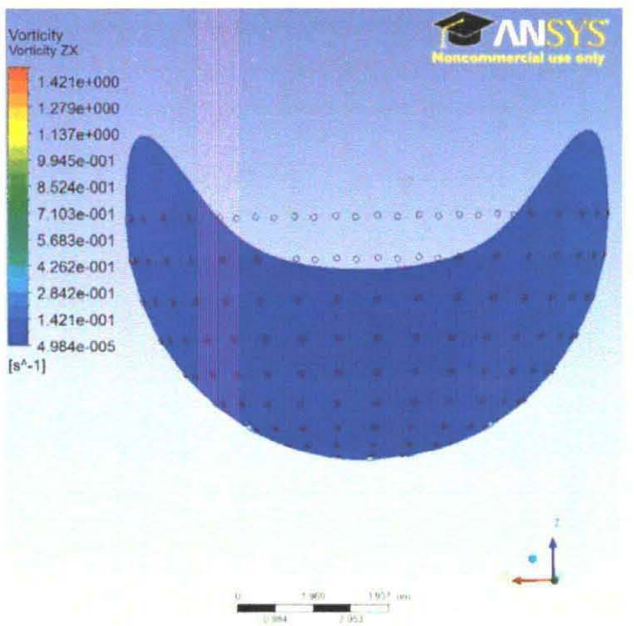

Figure 48. Vorticity Contours, $\mathrm{ZX}$ Plane, $\mathrm{t}=5.50 \mathrm{~s}$

The first fluid behavior investigated in the rigid, crater-shaped diaphragm tank is the presence of subsurface vortices in the tank. Figure 47 illustrates the output of the velocity vectors in the ZX plane at the $110^{\text {th }}$ timestep or $5.50 \mathrm{~s}$ into the simulation. It is noted that the velocity vectors are all linear in behavior and there is no indication of sub-surface vortices. Furthermore, there is no indication of sub-surface vortices forming at any time step in this simulation. This assertion is validated by considering the vorticity contours in the ZX plane.

Figure 48 illustrates the vorticity contours in the ZX plane at the $110^{\text {th }}$ time step. It is observed that the vorticity is nearly zero throughout the entire plane. Zero vorticity would imply no velocity curl in the tank and thus, no sub-surface vortices. However, the legend in the left-side of the figure indicates noticeably high vorticity at some location in the plane. Upon closer inspection, this high vorticity is observed along the hemispherical asperities. The details of this observation are presented later in the simulation analysis. 


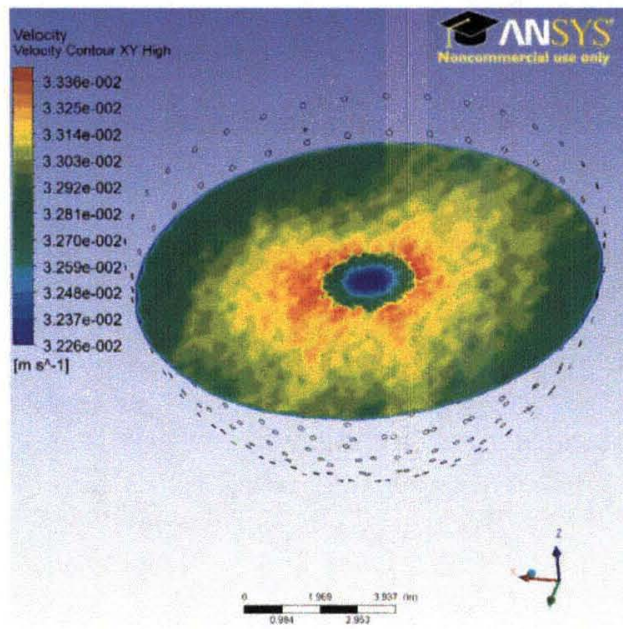

Figure 49. Velocity Contours, XY Plane, $t=5.50 \mathrm{~s}$

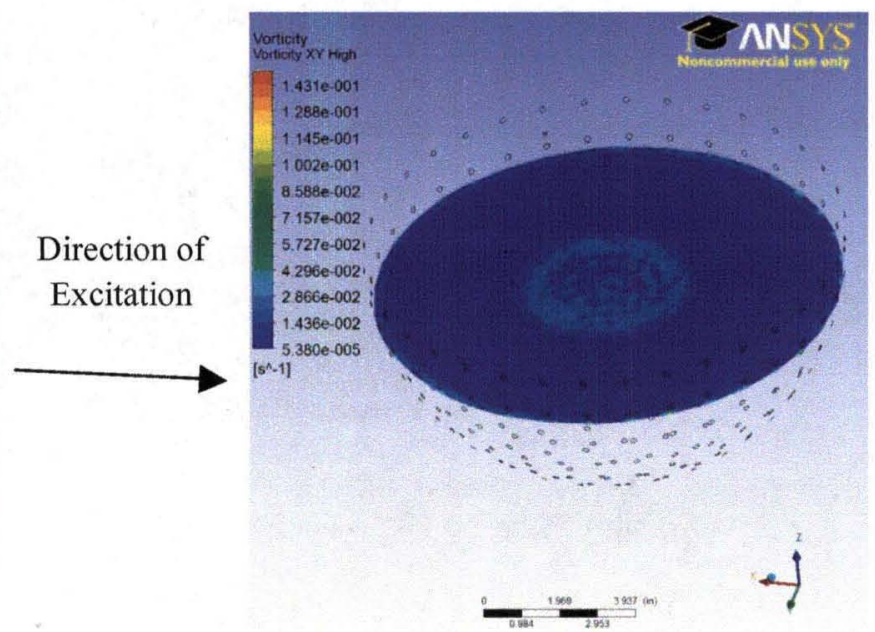

Figure 50. Vorticity Contours, XY Plane, $t=5.50 \mathrm{~s}$

Figures 49 and 50 are included in the analysis of the spherical asperities simply to remain consistent with the other analysis sets of the asperity simulations. There is nothing in Figure 48 to suggest vorticity in the upper volume of the tank but clarifying this point adds to the validity of the simulation. Figure 49 illustrates the velocity contours in the XY plane located at the plane tangent to the low point of the crater-shaped diaphragm. It is noted, as in other similar analysis, that the velocity is substantially lower in the middle of the tank in this plane. The velocity quickly increases just outside the middle of the tank although not to an extreme magnitude. This quick increase in velocity is enough to generate small amounts of vorticity illustrated in Figure 59.

The vorticity illustrated in Figure 48 is nothing significant, much like the vorticity illustrated in similar XY plane outputs. The magnitude of this vorticity is not great enough to generate the sub-surface vortices which are causes for concern. Again, it is noted that there is larger vorticity located in this plane. A closer analysis of this Figure indicates the larger vorticity is located along the outer edge of the plane along the asperities. The details of this observatio are presented in the following output.

It is initially concluded that the spherical asperities function as intended and eliminate the sub-surface vortices which were present in the asperity-free tank.

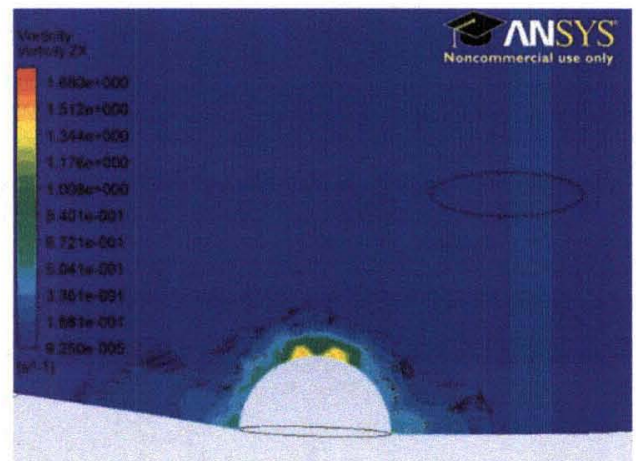

Figure 51. Vorticity Contours at Asperity, ZX Plane, $t=10.05 s$

Figure 51 is a closer look into the vorticity occurring at the hemispherical asperity. The highest vorticity is taking place directly over the center of the asperity. Noting the scale of the Figure, this vorticity spans less than $1 \mathrm{~mm}$ in either direction. With a vorticity of this low magnitude, there is no concern for the possibility of sub-surface vortices forming in the tank. Additionally, the vorticity further above the asperity is nearly zero confirming that the vorticity from the asperity is not propagating further into the tank resulting in undesirable fluid behavior. 


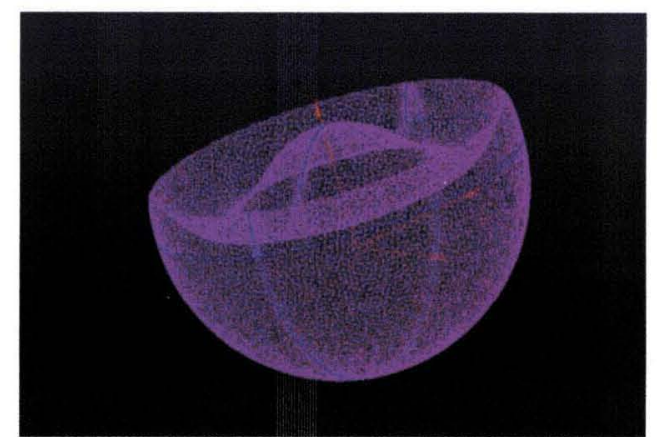

Figure 52. Meshed Tank with Rigid, Mountain-Shaped Diaphragm

Figure 52 illustrates the propellant tank implemented with a rigid, mountain-shaped diaphragm. Upon completing the frequency sweep, computational testing on this tank begins using an excitation frequency of $6.0 \mathrm{~Hz}$. No excitation in tangential force response was noted in the sweep until this frequency. Compared to the other metallic diaphragm simulations, this frequency is significantly higher. Using a high excitation frequency in the first computational simulation could pose problems in determining the first mode natural frequency of the system. Nonetheless, signal analysis using eigenvalues is used to determine the undamped and damped natural frequencies.

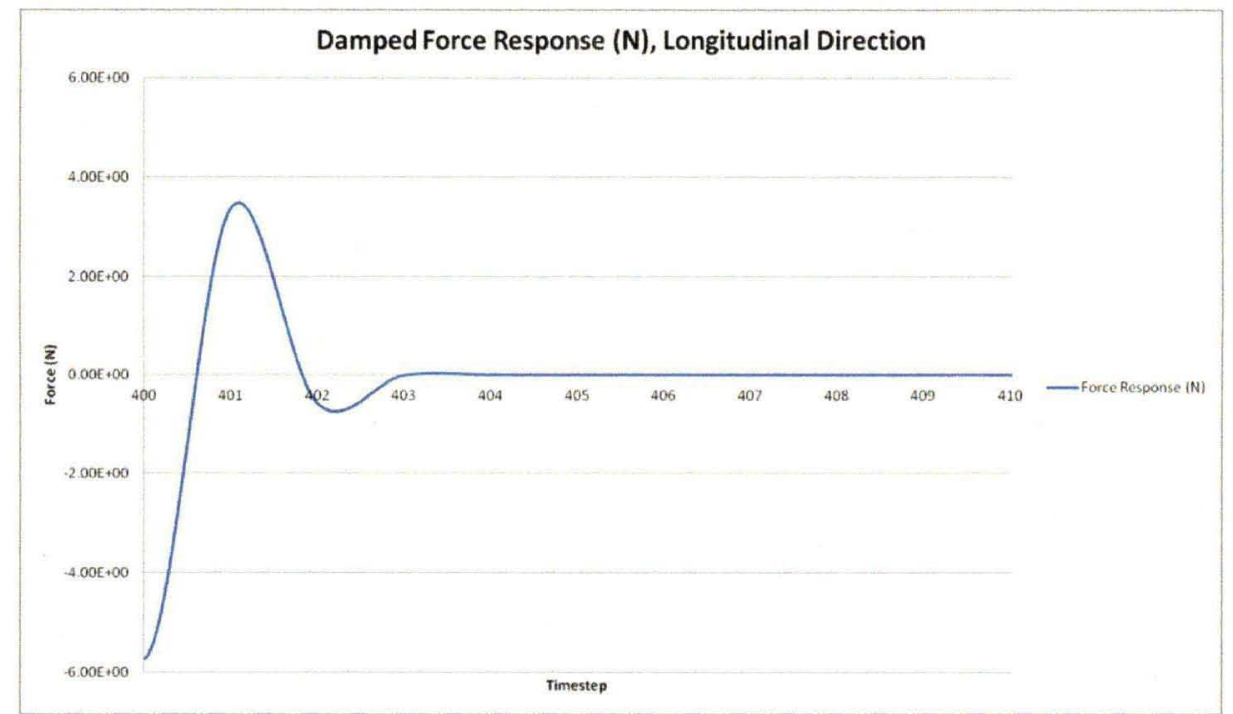

Figure 53. Rigid Mountain-Shaped Diaphragm Damped Force Response, Longitudinal Direction

The simulation for the rigid, mountain-shaped diaphragm tank is performed at an excitation frequency of $6.0 \mathrm{~Hz}$. Once the tank excitation ceases, the normal force response curve in the longitudinal direction follows Figure 53. Following the other metallic diaphragm simulations, the rigid, mountain-shaped diaphragm simulation dampens within four timesteps, or 0.2 seconds. With essentially one force response peak and no damping data, the simulation becomes difficult to analyze and extract the damping parameters. The data is input into MATLAB to solve the complex eigenvalue problem. Like the previous metallic diaphragm simulations, the reaction moment in the longitudinal direction is used as the second degree of freedom in the eigenvalue problem. The results of the eigenvalue algorithm are displayed in Table 6.

Table 6. Rigid, Mountain-Shaped Diaphragm Damping Results

\begin{tabular}{|c|c|}
\hline Parameter & Value \\
\hline$\omega_{\mathrm{n}}(\mathrm{rad} / \mathrm{s})$ & 24.3886 \\
\hline$\omega_{\mathrm{d}}(\mathrm{rad} / \mathrm{s})$ & 22.5174 \\
\hline$\zeta$ & 0.3841 \\
\hline
\end{tabular}


Table 6 presents the damping results taken from the 2-DOF eigenvalue problem computed by MATLAB for the rigid, mountain-shaped diaphragm tank. The undamped natural frequency of the system is $3.8816 \mathrm{~Hz}$, upon conversion, and the damped natural frequency of the system is $3.5838 \mathrm{~Hz}$. As suspected, these natural frequency values are significantly larger as compared to the natural frequency values of either the rigid, crater-shaped diaphragm tank without asperities or the tank with asperities. Therefore, it cannot be concluded if the natural frequency values calculated here are first mode natural frequencies.

MATLAB also generates the damping ratio, $\zeta$, of this system from the coupled, damped force response data. The value of 0.3841 is a useful parameter for alternative modeling of this system but like the rigid, cratershaped diaphragm system, it is not applicable in this part of the research at this point. However, due to the lack of data and peaks in the force response curve of Figure 62, it is not possible to obtain a curve-fit to this data to extract a damping ratio value, $\zeta_{\mathrm{F}}$. Therefore, the damping ratio of the force response curve cannot be found at this time.

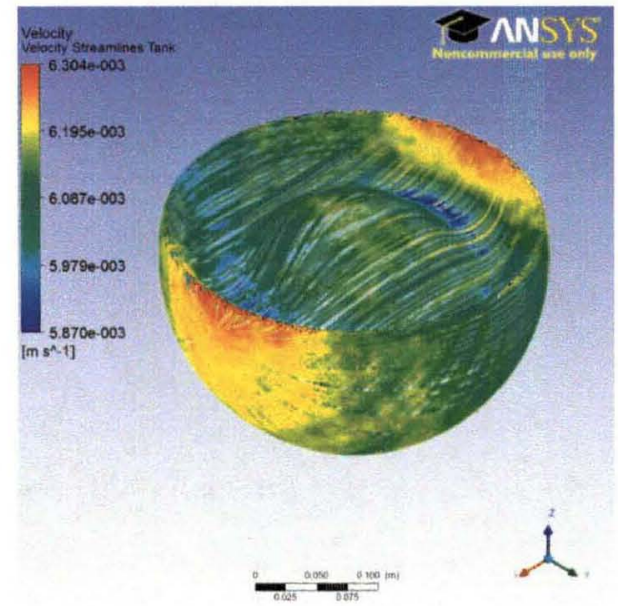

Figure 54. Velocity Surface Streamlines, Timestep 15

Following the other metallic diaphragm simulation results, it is important not only to investigate the damping parameters of the system, but also to explore the fluid behavior of the system from the CFX-Post outputs. Figure 54 illustrates the velocity surface streamlines during the excitation period of the system. The behavior of the fluid and the nature of the streamlines follow what is to be expected with the given simulation. The tank is being excited about the global x-axis (see the coordinate frame in the lower right corner of Figure 54). As such, it is expected that the locations of the zero-velocity streamlines are exactly where the blue streamlines are located. As the fluid moves along this streamline, it essentially comes to a stop in the location of the blue streamline because the diaphragm is blocking its movement. CFX does an excellent job of recognizing this and displaying it appropriately. Furthermore, it is interesting to note the location of the streamlines with the largest magnitude. Unfortunately, no explanation is readily available for this observation.

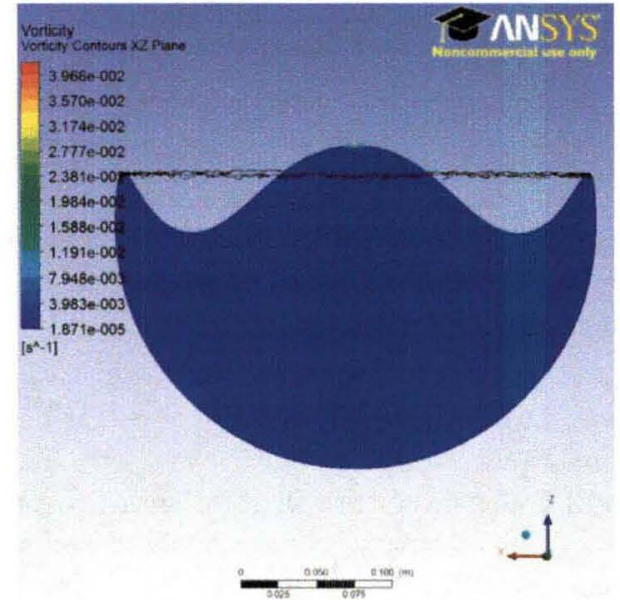

Figure 55. Vorticity, XZ Plane, Timestep 15 
It was noted in the rigid, crater-shaped diaphragm tank that sub-surface vortices occurred in the tank periodically during the simulation. With the addition of the asperities, the sub-surface vortices were eliminated. Careful analysis of the rigid, mountain-shaped diaphragm tank simulation has not shown the presence of any subsurface vortices. To verify this observation, Figure 55 illustrates the vorticity during a typical timestep in the excitation period. The only vorticity worth noting is the vorticity located at the top of the mountain-shaped diaphragm. Like the vorticity in the rigid, crater-shaped diaphragm tanks, this vorticity is not strong in magnitude and does not pose a threat to the stability of the propellant tank.

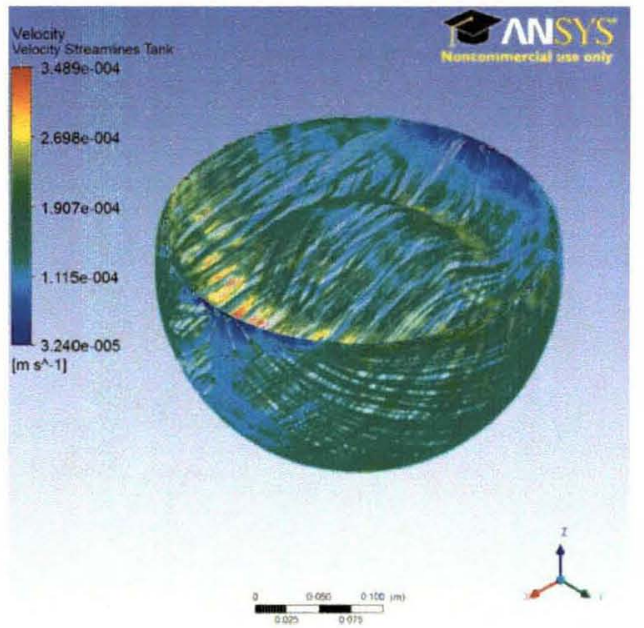

Figure 56. Velocity Surface Streamlines, Timestep 402

In keeping with the consistency of the results, the velocity streamlines are analyzed during the damping period. Figure 56 illustrates the velocity surface streamlines at the $402^{\text {nd }}$ timestep, or $0.1 \mathrm{~s}$ into damping. Referring to Figure 53, the fluid is nearly dampened by this timestep during damping and the velocity streamlines in Figure 56 verify that this is in fact the case. The majority of the velocity streamlines are on the order of magnitude $10^{-4}(\mathrm{~m} / \mathrm{s})$ with some even being $10^{-5}(\mathrm{~m} / \mathrm{s})$. Figure 65 is important because it illustrates the ability of CFX to accurately model the behavior of the fluid not only during the excitation period, but during the damping period as well.

\section{Flexible Diaphragms}

Currently, computational simulations for the flexible diaphragms are still being performed. When completed, the results will be documented in the M.S. thesis defense of the author.

\section{Conclusions}

\section{A. Free-Surface}

Table 7. Free-Surface Damping Parameters

\begin{tabular}{|c|c|}
\hline Parameter & Value \\
\hline$\omega_{\mathrm{n}}(\mathrm{Hz})$ & 1.7509 \\
\hline$\omega_{\mathrm{d}}(\mathrm{Hz})$ & 1.6907 \\
\hline$\zeta_{\mathrm{F}}$ & 0.07 \\
\hline
\end{tabular}

The results of the free-surface test contain acceptable values to be used as control values in this research investigation. The use of the FFT proved to be a quick and efficient means of determining the natural frequency of the system through the force response signal. Additionally, this research considers 2-D spectrograms an accurate alternative for determining the natural frequencies of a system through a force response signal. Lastly, the research investigation has shown that ANSYS CFX is capable of accurately modeling a free-surface spherical propellant tank. CFX-Post displayed the expected outputs for the free-surface simulation and provided useful data for accurately determining the damping parameters of the system. 


\section{B. Metallic Diaphragm}

The results of the metallic diaphragm testing generated excellent data and provided useful insight into the damping parameters and fluid behavior of such systems. Additionally, insight was gained into other fuel slosh parameters which could have had the ability to affect the system. Through computational testing, it was confirmed that gravity does not affect fluid behavior in propellant tank systems because the damping and other fluid parameters are based on mass and not weight of the system.

Past research has indicated the potential for spherical propellant tanks at a $100 \%$ fill-level to experience sub-surface inertial vortices. Although the simulations for the metallic diaphragm shapes do not incorporate a perfectly spherical tank, they are $100 \%$ full respectively and brought about speculation as to the occurrence of subsurface waves. Sub-surface waves were detected as hypothesized and appropriate methods to combat the vortices were designed and implemented into the computational tests. Both the linear and spherical asperities functioned as intended and eliminated the sub-surface vortices. However, through additional computational testing, it was deemed that the sub-surface vortices which occurred in these research simulations did not pose a serious threat to the propellant tank system. This is not to say that at the right frequency or tank geometry, the vortices could not pose a problem. For the purposes of this research investigation, the vortices were a non-factor. Thus, the use of asperities was foregone in the design of the rigid, mountain-shaped diaphragm tank.

Table 8. Table of Comparisons

\begin{tabular}{|c|c|c|c|}
\hline Model & $\omega_{\mathrm{n}}(\mathrm{Hz})$ & $\omega_{d}(H z)$ & $\alpha$ \\
\hline Free-Surface & 1.7509 & 1.6907 & N/A \\
\hline $\begin{array}{l}\text { Rigid Crater-Shaped } \\
\text { Diaphragm }\end{array}$ & 1.3834 & 1.2568 & $\begin{array}{c}-3.6321-7.8970 \mathrm{i} \\
-3.6321+7.8970 \mathrm{i} \\
-21.6988-5.6745 \mathrm{i} \\
-21.6988+5.6745 \mathrm{i} \\
\end{array}$ \\
\hline $\begin{array}{l}\text { Rigid Crater-Shaped } \\
\text { Diaphragm, Linear } \\
\text { Asperities, In-Plane }\end{array}$ & 1.3739 & 1.2983 & $\begin{array}{c}-2.9843-8.1008 \mathrm{i} \\
-2.9843+8.1008 \mathrm{i} \\
-19.6631-11.1052 \mathrm{i} \\
-19.6631+11.1052 \mathrm{i}\end{array}$ \\
\hline $\begin{array}{l}\text { Rigid Crater-Shaped } \\
\text { Diaphragm, Linear } \\
\text { Asperities, Out-of - } \\
\text { Plane }\end{array}$ & 1.3742 & 1.2798 & $\begin{array}{l}-3.1446-8.0412 \mathrm{i} \\
-3.1446+8.0412 \mathrm{i} \\
-20.6522-9.1278 \mathrm{i} \\
-20.6522+9.1278 \mathrm{i}\end{array}$ \\
\hline $\begin{array}{l}\text { Rigid Crater-Shaped } \\
\text { Diaphragm, Spherical } \\
\text { Asperities }\end{array}$ & 1.3754 & 1.2509 & $\begin{array}{c}-3.5933-7.8597 \mathrm{i} \\
-3.5933+7.8597 \mathrm{i} \\
-28.4588 \\
-17.8816\end{array}$ \\
\hline $\begin{array}{l}\text { Rigid Mountain- } \\
\text { Shaped Diaphragm }\end{array}$ & 3.8816 & $3.5838^{\circ}$ & $\begin{array}{l}-9.3687-22.5174 \mathrm{i} \\
-9.3687+22.5174 \mathrm{i} \\
-41.9104-40.4892 \mathrm{i} \\
-41.9104+40.4892 \mathrm{i}\end{array}$ \\
\hline
\end{tabular}

Table 8 compares the natural frequency values of the simulations and includes the alpha values from the eigenvalue problems. Focusing on the natural frequency values first, it is important to identify the undamped natural frequency values of the crater-shaped metallic diaphragm tanks are all approximately the same value of $1.37 \mathrm{~Hz}$. This is to be expected as the tanks were all essentially the same geometry and excited at the same excitation frequency of $2.2 \mathrm{~Hz}$. Additionally, the crater-shaped metallic diaphragms' undamped natural frequency value is less than that of the control, the free-surface tank. Furthermore, the damped natural frequency values of the crater-shaped metallic diaphragms are approximately the same values as well. The values do differ slightly, possibly a result of the orientation of the asperities as the fluid is attempting to dampen.

The natural frequency values of the rigid, mountain-shaped diaphragm must be used cautiously. These natural frequency values are clearly outside the apparent range of natural frequency values of the other models. It is 
possible that the first and/or second mode natural frequencies did not manifest themselves in the force response curve and the first natural frequency that was able to be identified was the third mode. This is not an uncommon occurrence in signal analysis and is the most feasible explanation as to why higher natural frequency values were obtained for this model.

Lastly, Table 8 indicates the alpha values for the models which were analyzed by the eigenvalue method. The alpha values are important values, not only for determining the natural frequencies and the damping ratio for the system, but the values are used to comment on the stability of the system. By considering the real part of the complex alpha, the stability of the system can be determined. The system is considered stable if the real part of alpha is negative. In all models, the real part of alpha is in fact negative, indicating that all models (except for the freesurface model) are stable.

\section{Flexible Diaphragm}

The conclusions of the flexible diaphragm simulation will be documented in the M.S. thesis defense of the author.

\section{Future Work}

In the coming months, the research investigation plans to move forward with the results obtained in this paper. The natural frequency values of the metallic diaphragm simulations need to be verified. Ideally, the use of 2D spectrograms as the comparison means is preferred but uncoupling the data may pose issues. Therefore, alternative signal analysis methods will be explored for use in verifying the natural frequencies of the metallic diaphragm simulations. Additionally, efforts will be made to determine the damping ratio of the force response curves, $\zeta_{\mathrm{F}}$, for highly damped simulations. This is a critical parameter for preliminary spacecraft design and one which must be obtained. Finally, the results of the flexible diaphragm simulation will be documented. The simulation will be analyzed and conclusions drawn as to the damping parameters and fluid behavior of the system.

Upon completing the follow-up work with the current simulations, new simulations will be introduced to the research. A computational simulation modeling tank excitation while propellant is depleting from the tank is desired as this would simulate a true spacecraft fuel slosh scenario. Additional fuel slosh scenarios are desired to be modeled such as spin-up and spin-down maneuvers which could potentially induce unsteady fluid motion within the tank as well as simulations considering turbulence within the fluid.

\section{Acknowledgments}

The authors of this paper would like to thank ATK/PSI for the use of their diaphragm in this research investigation. Thank you to Embry-Riddle Aeronautical University (ERAU) for providing the computational resources and facilities. The authors would also like to thank Keith Schlee of Orbital Sciences for his guidance throughout this research. Thank you especially to Dr. Luis San Andres of Texas A\&M University for his assistance and lecture materials regarding MDOF viscous damped systems. Thank you to Dr. Ilteris Demirkiran of ERAU for assistance in developing MATLAB algorithms used for numerical analysis. Thank you to Dr. Frank Radosta from ERAU for guidance during numerical analysis. Lastly, the authors would like to thank Dhawal Leuva from Embry-Riddle Aeronautical University for his assistance while developing computational simulations for this research.

\section{References}

${ }^{1}$ Rao, S.S., Mechanical Vibrations. Upper Saddle River, New Jersey: Pearson Prentice Hall, 2004. Print.

${ }^{2}$ Fourier Transforms \& the Frequency Domain. http://astro.berkeley.edu/ jrg/ngst/fft/fourier.html. Accessed February 15, 2012.

${ }^{3}$ San Andrés, L., Modal Analysis of MDOF Systems with Viscous Damping. MEEN 617 HD 11, Texas A\&M University, 2008.

${ }^{4}$ CATIA V5R20, 2009, Dassault Systemes, http://www.3ds.com/catia. 
${ }^{5}$ ANSYS 13.0, 2011, ANSYS Inc, http://www.ansys.com.

${ }^{6}$ Pointwise V16.04, 2011, Pointwise Inc, http://www.pointwise.com.

${ }^{7}$ Hubert, C., Behavior of Spinning Space Vehicles with Onboard Liquids, NASA/Kennedy Space Center NAS10-02016, 2003.

${ }^{8}$ Verlag, C. H., Handbook of Space Technology, United Kingdom: John Wiley \& Sons, 2009. Online.

${ }^{9}$ Lenahen, B., Sances, D. Gangadharan, S., Accurately Correlating Experimental and Computational Spacecraft Fuel Slosh Models Using Diaphragm-Implemented Propellant Tanks, 34th Annual AAS Guidance and Control Conference, American Astronautical Society, AAS 11-063, Breckenridge, Colorado, 2011.

${ }^{10}$ ANSYS, Inc., ANSYS CFX Tutorials. USA. Release 12.1, November, 2009. PDF

${ }^{11}$ The Engineering Toolbox., http://www.engineeringtoolbox.com/liquids-densities-d 743.html. Accessed August 2010.

${ }^{12}$ Vorticity and Potential Vorticity. http://www.princeton.edu/ gkv/geoturb/turbch.pdf. Accessed. August $\underline{2011}$. 